\title{
Smart homing guidance strategy with control saturation against a cooperative target-defender team
}

\author{
GUO Hang*, FU Wenxing, FU Bin, CHEN Kang, and YAN Jie
}

School of Astronautics, Northwestern Polytechnical University, Xi' an 710072, China

\begin{abstract}
A smart homing guidance strategy with control saturation against a target-defender team is derived. It is noteworthy that a cooperative strategy of the target-defender team is applied, which has been proved more challenging for the homing guidance. The defender missile is launched by the target and guided by a cooperative augmented proportional navigation (APN). At the same time, the target performs a one-switch maneuver to cooperate and minimize the defender's acceleration requirement. The problem is analyzed for arbitrary-order linear dynamics of the agents in the linearized form but validated by the mathematical simulations by using nonlinear kinematics. The perfect information of three agents' states is assumed. Then, a method to deal with the target-defender team is proposed. It contains a combined performance index penalizing the miss distance relative to the target and energy consumption in the whole duration. Besides, the specific miss distance related to the defender is regarded as an inequality constraint. An analytical solution for the smart guidance strategy against the APN guided defender is derived. Meanwhile, the control saturations are introduced to get more realistic and reasonable insights to this practical target-missile-defender problem. A simple but effective iterative searching technique is proposed to determine the saturation time points. The solution provides an optimal homing strategy to evade the defender with a specific miss distance and intercept the target with the minimum miss distance in the minimum energy manner. Nonlinear two-dimensional simulation results are used to validate the theoretical analysis. By comparison with the optimal differential game guidance (ODGG) and the combined minimum effort guidance (CMEG), the superiority of this smart guidance strategy is concluded.
\end{abstract}

Keywords: smart homing guidance, control saturation, targetmissile-defender, iterative searching technique.

DOI: $10.21629 / J S E E .2019 .02 .15$

\section{Introduction}

The three agents' pursuit-evasion problem [1] has attracted great interest in recent years, which is basically

\footnotetext{
Manuscript received May 29, 2018

*Corresponding author.

This work was supported by the National Natural Science Foundation of China (91216104; 61503302).
}

expressed as the target-missile-defender (TMD) scenario: an evading target (T), a homing missile (M) and a defending missile (D).

Traceably, this TMD problem derives from the traditional pursuit-evasion problem, and the optimal control theory [2,3], the differential game theory [4-6], the model predictive control $[7,8]$, etc. are validated to be effective methods. Recently, a great number of studies over the years have attacked the TMD problem. A multiple model adaptive control (MMAC) approach was proposed in [9]. It estimates the guidance law and detailed parameters of the homing missile and then a cooperative guidance strategy for the target-defender team is derived. Consequently, the homing missile is intercepted by the defender and the target escapes safely. An active target defense differential game problem was dealt with in [10], where the closedform analytical solution was provided. In addition, the set of initial conditions under which the target is capable of escaping is provided. In [11], cooperative nonlinear guidance strategies for the target and missile by the sliding-mode control technique were developed, which were validated to possess the superior performance compared with existing classical guidance laws. An optimal guidance was derived in [12], for the target to cooperate with the defender and evade successfully. In [13], weapon-target-allocation algorithms were proposed in an interception scenario between multiple targets, missiles and defenders. The algorithms provide the close-to-optimal performance with a significant improvement on the computational efficiency. Other studies [14-18] about the cooperative guidance between the target and the defender also demonstrate the cooperative team's superiority than the residual single agent. Motivated by this challenging situation for the homing missile, a smart guidance strategy is developed from the homing missile point of view.

With regarding to the homing missile, several studies focus on the optimal guidance strategy, but the research is conducted in a relatively simple and ideal sense. For exam- 
ple, in [19], algebraic conditions for the pursuer to capture the target while evading the defender were given, but three agents were assumed ideal dynamics, linear kinematics and executing bounded control constantly. A differential game guidance strategy for the homing missile was derived in [20]. However, the solutions for three agents are all conservative and final results depend on their maneuverability. In addition, the analytical solutions are difficult to obtain when the agents' dynamics are nonideal. Another optimal guidance strategy of the homing missile was derived from the perspective of the minimum efforts in [21]. Nevertheless, this combined minimum efforts guidance (CMEG) algorithm for the homing missile is developed against a separated team between the target and the defender. Besides, the target's maneuver is assumed constant. More importantly, to evade the defender with the specific miss distance, the time constant of the defender's dynamics is assumed much larger than that of the missile's dynamics, which is not aligned with the practical situations.

In summary, the research on the guidance of the homing missile against the cooperative target and the defender will be pretty challenging and promising [22-34]. Synthesizing all the research mentioned above, the main contributions of this paper could be concluded in the following aspects:

(i) A smart guidance strategy for the homing missile is derived. It deals with a cooperative team between the target and the defender. These two agents conduct the cooperative strategy [18] simultaneously.

(ii) The neoteric means to tackle two miss distances is proposed, which is in accordance with practical situations and leads to more reasonable and convincing conclusions. The miss distance between $\mathrm{M}$ and $\mathrm{T}$ is incorporated into the performance index to guarantee precise interception for the target, while the miss distance between $\mathrm{M}$ and $\mathrm{D}$ is treated as the inequality constraint.

(iii) Nonlinear factors, including the control saturations, are introduced into the integral guidance problem. Besides, an efficient iterative searching technique is proposed to determine the saturation time points. Consequently, compared with the optimal differential game guidance (ODGG) [20] and the CMEG [21], the proposed guidance algorithm expresses superior performance in practical situations.

The structure of this paper is organized as follows. The model of the TMD problem is presented in Section 2. The guidance problem is formulated with the proper performance index. Then, the smart guidance law is derived without control saturations firstly in Section 3, followed by further implementation for the guidance strategy with control saturations in Section 4. The guidance algorithm is evalu- ated by numerical simulation in Section 5 and final conclusions are drawn in Section 6.

\section{TMD guidance problem formulation}

A planar three-body engagement scenario is considered, where the missile (M) homes to the target (T) and the defender (D) tries to intercept the missile, as shown in Fig. 1. It assumes that three agents are constant speed points massed with arbitrary linear dynamics.

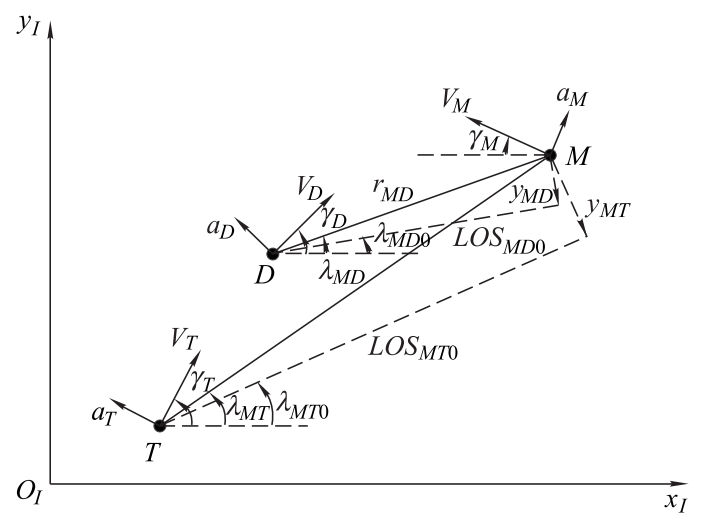

Fig. 1 Planar three-body engagement scenario

\subsection{Nonlinear kinematics}

In the sequel, the interception between the homing missile and the evading target will be denoted as $M T$, while that between the homing missile and the defender missile will be denoted as $M D$.

$Z_{M T}(t)$ The terminal engagement time of the targetmissile and the missile-defender can be approximated by the following two equations respectively:

$$
\begin{aligned}
t_{f M T} & =r_{M T}(0) / V_{c M T} \\
t_{f M D} & =r_{M D}(0) / V_{c M D}
\end{aligned}
$$

where $r_{i j}, V_{c i j}$ and $t_{f i j}$ denote the relative ranges, closing speeds and terminal engagement time with $i, j \in$ $\{M, T, D\}, i \neq j$, respectively.

Understandably, it assumes that the $M D$ engagement terminated before the $M T$ engagement so that the mission of the defender is meaningful, that is, $t_{f M T}>t_{f M D}$. Then the corresponding time-to-goes $t_{g o M T}$ and $t_{\text {goMD }}$ could be expressed as follows:

$$
\begin{gathered}
t_{g o M T}=t_{f M T}-t \\
t_{g o M D}=t_{f M D}-t .
\end{gathered}
$$

The nonlinear relative kinematics of the $M T$ and the $M D$ engagement could be formulated by

$$
\begin{gathered}
\dot{r}_{M T}=-V_{M} \cos \left(\gamma_{M}+\lambda_{M T}\right)-V_{T} \cos \left(\gamma_{T}-\lambda_{M T}\right) \\
\dot{\lambda}_{M T}=\left[V_{M} \sin \left(\gamma_{M}+\lambda_{M T}\right)-V_{T} \sin \left(\gamma_{T}-\lambda_{M T}\right)\right] / r_{M T}
\end{gathered}
$$




$$
\begin{gathered}
\dot{r}_{M D}=-V_{M} \cos \left(\gamma_{M}+\lambda_{M D}\right)-V_{D} \cos \left(\gamma_{D}-\lambda_{M D}\right) \\
\dot{\lambda}_{M D}=\left[V_{M} \sin \left(\gamma_{M}+\lambda_{M D}\right)-V_{D} \sin \left(\gamma_{D}-\lambda_{M D}\right)\right] / r_{M D}
\end{gathered}
$$

where $V_{i}$ and $\gamma_{i}$ represent the velocity and the flight path angle respectively with $i \in\{M, T, D\}, \lambda_{M T}$ is the angle of line-of-sight (LOS) from $T$ to $M$, and $\lambda_{M D}$ denotes the angle of line of sight from $D$ to $M$.

In the whole engagement duration, the three agents' flight path angles evolve according to the following equation:

$$
\gamma_{i}=a_{i} / V_{i}, \quad i=\{M, T, D\}
$$

where $a_{i}$ denotes the vehicle's turning acceleration.

Additionally, the dynamics of each vehicle is formulated by arbitrary-order linear equations:

$$
\left\{\begin{array}{l}
\dot{\boldsymbol{x}}_{i}=A_{i} \boldsymbol{x}_{i}+b_{i} \boldsymbol{u}_{i}^{\prime} \\
a_{i}=c_{i} \boldsymbol{x}_{i}+d_{i} \boldsymbol{u}_{i}^{\prime}
\end{array}\right.
$$

where $\boldsymbol{x}_{i}(i=\{M, T, D\})$, is the state vector with $\operatorname{dim}\left(\boldsymbol{x}_{i}\right)=n_{i}$ and $\boldsymbol{u}_{i}^{\prime}$ denotes its turning acceleration command.

\subsection{Linearized kinematics}

During the whole engagement with three entities, the endgame phase is dealt with. Therefore, it assumes that the flight scenario evolves nearly two nominal collision triangles, and therefore the kinematics linearization can be performed around the initial collision triangles.

The relative displacements normal to $\operatorname{LOS}_{M T}$ and $\operatorname{LOS}_{M D}$ are selected as the state elements of the linearized model. Besides, each agent's acceleration normal to the LOS is denoted as $a_{i N}$ and thus

$$
\left\{\begin{array}{c}
\boldsymbol{a}_{M N}=a_{M} \cos \left(\gamma_{M 0}+\lambda_{M T 0}\right) \\
\quad=c_{M} \boldsymbol{x}_{M} \cos \left(\gamma_{M 0}+\lambda_{M T 0}\right)+d_{M} \boldsymbol{u}_{M}, \\
\boldsymbol{a}_{T N}=a_{T} \cos \left(\gamma_{T 0}-\lambda_{M T 0}\right) \\
\quad=c_{T} \boldsymbol{x}_{T} \cos \left(\gamma_{T 0}-\lambda_{M T 0}\right)+d_{T} \boldsymbol{u}_{T}, \\
\boldsymbol{a}_{D N}=a_{D} \cos \left(\gamma_{D 0}-\lambda_{M D 0}\right) \\
\quad=c_{D} \boldsymbol{x}_{D} \cos \left(\gamma_{D 0}-\lambda_{M D 0}\right)+d_{D} \boldsymbol{u}_{D}
\end{array}\right.
$$

where $\boldsymbol{u}_{i}(i \in\{T, M, D\})$ is each agent's acceleration command normal to the corresponding LOS, can be which expressed as follows:

$$
\left\{\begin{array}{l}
\boldsymbol{u}_{M}=\boldsymbol{u}_{M}^{\prime} \cos \left(\gamma_{M 0}+\lambda_{M T 0}\right) \\
\boldsymbol{u}_{T}=\boldsymbol{u}_{T}^{\prime} \cos \left(\gamma_{T 0}-\lambda_{M T 0}\right) \\
\boldsymbol{u}_{D}=\boldsymbol{u}_{D}^{\prime} \cos \left(\gamma_{D 0}-\lambda_{M D 0}\right)
\end{array} .\right.
$$

The state vector of the linearized problem is

$$
\boldsymbol{x}=\left[\begin{array}{lll}
\boldsymbol{x}_{M T}^{\mathrm{T}} & \boldsymbol{x}_{M D}^{\mathrm{T}} & \boldsymbol{x}_{M}^{\mathrm{T}}
\end{array}\right]^{\mathrm{T}}
$$

where

$$
\begin{aligned}
\boldsymbol{x}_{M T} & =\left[\begin{array}{lll}
y_{M T} & \dot{y}_{M T} & \boldsymbol{x}_{T}^{\mathrm{T}}
\end{array}\right]^{\mathrm{T}} \\
\boldsymbol{x}_{M D} & =\left[\begin{array}{lll}
y_{M D} & \dot{y}_{M D} & \boldsymbol{x}_{D}^{\mathrm{T}}
\end{array}\right]^{\mathrm{T}}
\end{aligned}
$$

and $\operatorname{dim}(\boldsymbol{x})=4+n_{M}+n_{T}+n_{D}$. The linearized equations of motion could be described as follows:

$$
\dot{\boldsymbol{x}}=\boldsymbol{A} \boldsymbol{x}+\boldsymbol{B}_{T} \boldsymbol{u}_{T}+\boldsymbol{B}_{D} \boldsymbol{u}_{D}+\boldsymbol{B}_{M} \boldsymbol{u}_{M}
$$

where

$$
\begin{aligned}
& \boldsymbol{A}=\left[\begin{array}{ccc}
\boldsymbol{A}_{M T} & \mathbf{0}_{\left(n_{T}+2\right) \times\left(n_{D}+2\right)} & \boldsymbol{E}_{M T} \\
\mathbf{0}_{\left(n_{D}+2\right) \times\left(n_{T}+2\right)} & \boldsymbol{A}_{M D} & \boldsymbol{E}_{M D} \\
\mathbf{0}_{n_{M} \times\left(n_{T}+2\right)} & \mathbf{0}_{n_{M} \times\left(n_{D}+2\right)} & \boldsymbol{A}_{M}
\end{array}\right] \\
& \boldsymbol{B}_{T}=\left[\begin{array}{c}
\boldsymbol{B}_{M T} \\
\mathbf{0}_{\left(n_{D}+2\right) \times 1} \\
\mathbf{0}_{n_{M} \times 1}
\end{array}\right] \\
& \boldsymbol{B}_{D}=\left[\begin{array}{c}
\mathbf{0}_{\left(n_{T}+2\right) \times 1} \\
\boldsymbol{B}_{M D} \\
\mathbf{0}_{n_{M} \times 1}
\end{array}\right]
\end{aligned}
$$

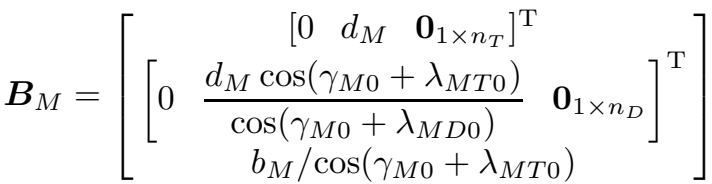

and

$$
\begin{aligned}
& \boldsymbol{A}_{M T}=\left[\begin{array}{ccc}
0 & 1 & \mathbf{0}_{1 \times n_{T}} \\
0 & 0 & -c_{T} \cos \left(\gamma_{T 0}-\lambda_{M T 0}\right) \\
\mathbf{0}_{n_{T} \times 1} & \mathbf{0}_{n_{T} \times 1} & A_{T}
\end{array}\right] \\
& \boldsymbol{B}_{M T}=\left[\begin{array}{c}
0 \\
-d_{T} \\
b_{T} / \cos \left(\gamma_{T 0}-\lambda_{M T 0}\right)
\end{array}\right] \\
& \boldsymbol{A}_{M D}=\left[\begin{array}{ccc}
0 & 1 & \mathbf{0}_{1 \times n_{D}} \\
0 & 0 & -c_{D} \cos \left(\gamma_{D 0}-\lambda_{M D 0}\right) \\
\mathbf{0}_{n_{D} \times 1} & \mathbf{0}_{n_{D} \times 1} & A_{D}
\end{array}\right] \\
& \boldsymbol{B}_{M D}=\left[\begin{array}{c}
0 \\
-d_{D} \\
b_{D} / \cos \left(\gamma_{D 0}-\lambda_{M D 0}\right)
\end{array}\right] \\
& \boldsymbol{E}_{M T}=\left[\begin{array}{c}
\mathbf{0}_{1 \times n_{M}} \\
c_{M} \cos \left(\gamma_{M 0}+\lambda_{M T 0}\right) \\
\mathbf{0}_{n_{T} \times n_{M}}
\end{array}\right] \\
& \boldsymbol{E}_{M D}=\left[\begin{array}{c}
\mathbf{0}_{1 \times n_{M}} \\
c_{M} \cos \left(\gamma_{M 0}+\lambda_{M D 0}\right) \\
\mathbf{0}_{n_{D} \times n_{M}}
\end{array}\right] \text {. }
\end{aligned}
$$

\section{Guidance algorithm for a smart homing missile without control saturations}

In this paper, the success of the homing missile should be 
defined by the following two conditions:

(i) The miss distance $y_{M D}\left(t_{f M D}\right)$ is larger than $M_{M D}$ (the lethality radius of the defender).

(ii) The miss distance $y_{M T}\left(t_{f M T}\right)$ is as small as possible (at least smaller than the lethality radius of the homing missile).

The control effort is chosen as the quadratic integral of the guidance command. Usually, $y_{M T}\left(t_{f M T}\right)$ would be enlarged when the missile maneuveres evade the defender. Therefore, after the evasion process, the minimum $y_{M T}\left(t_{f M T}\right)$ becomes more important than the energy consumption. As a result, the $y_{M T}\left(t_{f M T}\right)$ is incorporated into the performance index, which is formulated by

$$
J=\frac{a}{2} y_{M T}^{2}\left(t_{f M T}\right)+\frac{1}{2} \int_{t_{0}}^{t_{f M T}} \boldsymbol{u}_{M}^{2}(t) \mathrm{d} t
$$

subject to

$$
\left|y_{M D}\left(t_{f M D}\right)\right| \geqslant M_{M D}
$$

It assumes that the target-defender team applies a cooperative guidance strategy [18]. The target conducts a oneswitch maneuver by the maximum acceleration from one side to another. The switch time point depends on the initial engagement scenario. The defender applies an optimal interception guidance law, which takes the homing missile's acceleration and dynamics properties into consideration. In [18], the switch time for the target could be described as follows:

$$
t_{s w}=\underset{t_{s w} \in\left[t_{k}, t_{f}\right]}{\arg \min }\left[\sum_{j=1}^{s} \mu_{k}^{j} Z_{d m}^{j}\right]
$$

and the guidance law for the defender could be expressed as follows:

$$
u_{D}^{*}(t)=\frac{N_{\mathrm{COGL}}^{\prime} Z_{d m}(t)}{\left(t_{g o}^{d m}\right)^{2}} .
$$

However, to obtain the analytical solution, the cooperative guidance strategy of the target-defender team is required to be simplified. Therefore, the switch time for the target is set as follows:

$$
t_{s w}=\frac{1}{2} t_{g o M D}
$$

and the guidance law of the defender is degenerated to the augmented proportional navigation (APN) guidance law:

$$
u_{D}(t)=\frac{N_{\mathrm{APN}}^{\prime} \cdot Z_{M D}(t)}{\left(t_{g o M D}\right)^{2}}
$$

where

$$
\begin{gathered}
Z_{M D}(t)=y_{M D}+\dot{y}_{M D} \cdot t_{g o M D}+ \\
\frac{1}{2} a_{M} \cos \left(\gamma_{M}+\lambda_{M D}\right) t_{g o M D}^{2}
\end{gathered}
$$

and $N_{\mathrm{APN}}^{\prime}$ is a constant gain.

\subsection{Reduced-order optimal problem formulation}

The acceleration command of the defender could be marked as follows:

$$
u_{D}(t)=\boldsymbol{F}_{D}(t) \boldsymbol{x}
$$

then (16) becomes

$$
\begin{gathered}
\dot{\boldsymbol{x}}=\left(\boldsymbol{A}+\boldsymbol{B}_{D} \boldsymbol{F}_{D}(t)\right) \boldsymbol{x}+\boldsymbol{B}_{T} \boldsymbol{u}_{T}(t)+\boldsymbol{B}_{M} \boldsymbol{u}_{M}(t) \triangleq \\
\boldsymbol{A}_{T D} \boldsymbol{x}+\boldsymbol{B}_{T} \boldsymbol{u}_{T}+\boldsymbol{B}_{M} \boldsymbol{u}_{M}
\end{gathered}
$$

with the initial condition

$$
\boldsymbol{x}\left(t_{0}\right)=\boldsymbol{x}_{0}
$$

To obtain the optimal guidance algorithm, the zeroeffort miss transformation is introduced to reduce the dimension of the state vector:

$$
\begin{gathered}
Z_{M T}(t)=\boldsymbol{d}_{M T}^{\mathrm{T}} \boldsymbol{\Phi}_{T D}\left(t_{f M T}, t\right) \boldsymbol{x}(t)+ \\
\int_{t}^{t_{f M T}} \boldsymbol{d}_{M T}^{\mathrm{T}} \boldsymbol{\Phi}_{T D}\left(t_{f M T}, \tau\right) \boldsymbol{B}_{T} \boldsymbol{u}_{T} \mathrm{~d} \tau \\
Z_{M D}(t)=\boldsymbol{d}_{M D}^{\mathrm{T}} \boldsymbol{\Phi}_{T D}\left(t_{f M D}, t\right) \boldsymbol{x}(t)
\end{gathered}
$$

where

$$
\left\{\begin{array}{l}
\boldsymbol{d}_{M T}=\left[\begin{array}{lllllll}
1 & 0 & 0 & 0 & 0 & 0 & 0
\end{array}\right]^{\mathrm{T}} \\
\boldsymbol{d}_{M D}=\left[\begin{array}{lllllll}
0 & 0 & 0 & 1 & 0 & 0 & 0
\end{array}\right]^{\mathrm{T}}
\end{array}\right.
$$

and $\boldsymbol{\Phi}_{T D}\left(t_{f M T}, t\right)$ and $\boldsymbol{\Phi}_{T D}\left(t_{f M D}, t\right)$ are the corresponding state transition matrices.

Meanwhile, the two zero-effort miss distances satisfy

$$
\left\{\begin{array}{l}
\dot{Z}_{M T}=d_{M T}^{\mathrm{T}} \boldsymbol{\Phi}_{T D}\left(t_{f M T}, t\right) B_{M} \boldsymbol{u}_{M} \triangleq \alpha(t) \boldsymbol{u}_{M} \\
\dot{Z}_{M D}=d_{M D}^{\mathrm{T}} \boldsymbol{\Phi}_{T D}\left(t_{f M D}, t\right) B_{M} \boldsymbol{u}_{M} \triangleq \beta(t) \boldsymbol{u}_{M}
\end{array} .\right.
$$

Besides, for $t>t_{f M D}$, to keep the overall mathematical problem continuous, it assumes that

$$
Z_{M D}(t) \equiv Z_{M D}\left(t_{f M D}\right), \quad \boldsymbol{\beta}(t) \equiv 0 .
$$

With regarding to the reduced-order system (40), the performance index $J$ becomes

$$
J=\frac{a}{2} Z_{M T}^{2}\left(t_{f M T}\right)+\frac{1}{2} \int_{t_{0}}^{t_{f M T}} \boldsymbol{u}_{M}^{2} \mathrm{~d} t
$$

Then the Hamilton function could be formulated as follows:

$$
H=\frac{1}{2} \boldsymbol{u}_{M}^{2}+\lambda_{1} \boldsymbol{\alpha}(t) \boldsymbol{u}_{M}+\lambda_{2} \boldsymbol{\beta}(t) \boldsymbol{u}_{M}
$$


Therefore, the costate equations could be given as follows:

$$
\left\{\begin{array}{l}
\dot{\lambda}_{1}=-\frac{\partial H}{\partial Z_{M T}(t)}=0 \\
\dot{\lambda}_{2}=-\frac{\partial H}{\partial Z_{M D}(t)}=0
\end{array}\right.
$$

with the following transversality condition:

$$
\left\{\begin{array}{l}
\lambda_{1}\left(t_{f M T}\right)=\frac{\partial J}{\partial Z_{M T}\left(t_{f M T}\right)}=a Z_{M T}\left(t_{f M T}\right) \\
\lambda_{2}\left(t_{f M T}\right)=\frac{\partial J}{\partial Z_{M D}\left(t_{f M T}\right)}=0
\end{array} .\right.
$$

Thus the costate vector could be obtained that

$$
\left\{\begin{array}{l}
\lambda_{1}(t)=a Z_{M T}\left(t_{f M T}\right) \\
\lambda_{2}(t)=0
\end{array} .\right.
$$

According to the control equation, the optimal control satisfies

$$
\frac{\partial H}{\partial \boldsymbol{u}_{M}^{*}}=\boldsymbol{u}_{M}^{*}+\lambda_{1} \boldsymbol{\alpha}(t)+\lambda_{2} \boldsymbol{\beta}(t)=0,
$$

then the open loop optimal control could be given as follows:

$$
\boldsymbol{u}_{M}^{*}=-a Z_{M T}\left(t_{f M T}\right) \boldsymbol{\alpha}(t) .
$$

Due to (40), the zero-effort miss $Z_{M T}(t)$ satisfies

$$
Z_{M T}(t)+\int_{t}^{t_{f M T}} \boldsymbol{\alpha}(\tau) \boldsymbol{u}_{M} \mathrm{~d} \tau=Z_{M T}\left(t_{f M T}\right) .
$$

Once the optimal control $\boldsymbol{u}_{M}^{*}$ is applied,

$$
Z_{M T}\left(t_{f M T}\right)=\frac{Z_{M T}(t)}{1+a \int_{t}^{t_{f M T}} \boldsymbol{\alpha}^{2}(\tau) \mathrm{d} \tau} .
$$

Therefore, the closed loop optimal control could be expressed as follows:

$$
\boldsymbol{u}_{M}^{*}=\frac{-Z_{M T}(t) \boldsymbol{\alpha}(t)}{1 / a+\int_{t}^{t_{f M T}} \boldsymbol{\alpha}^{2}(\tau) \mathrm{d} \tau} .
$$

\subsection{Measurements on the inequality constraint}

Notice that the derivation process in Section A does not take the inequality constraint (28) into account. With regarding to the zero-effort miss $Z_{M D}(t)$, it satisfies

$$
Z_{M D}(t)+\int_{t}^{t_{f M D}} \boldsymbol{\beta}(\tau) \boldsymbol{u}_{M} \mathrm{~d} \tau=Z_{M D}\left(t_{f M D}\right) .
$$

When the closed loop optimal control (51) is applied, it could be acquired that

$$
Z_{M D}\left(t_{f M D}\right)=
$$

$$
Z_{M D}\left(t_{0}\right)-\frac{Z_{M T}\left(t_{0}\right) \int_{t_{0}}^{t_{f M D}} \boldsymbol{\alpha}(\tau) \boldsymbol{\beta}(\tau) \mathrm{d} \tau}{1 / a+\int_{t_{0}}^{t_{f M D}} \boldsymbol{\alpha}^{2}(\tau) \mathrm{d} \tau} .
$$

Under this circumstance, if

$$
\left|Z_{M D}\left(t_{f M D}\right)\right| \geqslant M_{M D}
$$

the closed loop optimal control $\boldsymbol{u}_{M}^{*}$ (51) will be optimal. If (54) is not satisfied, the variable $Z_{M T}\left(t_{f M T}\right)$ will be calculated from the inequality constraint point of view. Therefore,

$$
\begin{gathered}
Z_{M D}\left(t_{f M D}\right)= \\
Z_{M D}(t)+\int_{t}^{t_{f M D}}\left[-a Z_{M T}\left(t_{f M T}\right)\right] \boldsymbol{\alpha}(\tau) \boldsymbol{\beta}(\tau) \mathrm{d} \tau .
\end{gathered}
$$

Then the closed loop optimal control will be

$$
\boldsymbol{u}_{M}^{*}=\frac{\left(Z_{M D}\left(t_{f M D}\right)-Z_{M D}(t)\right) \boldsymbol{\alpha}(t)}{\int_{t}^{t_{f M D}} \boldsymbol{\alpha}(\tau) \boldsymbol{\beta}(\tau) \mathrm{d} \tau} .
$$

Substituting (56) into (42), the performance index turns into

$$
J=\frac{\left(Z_{M D}\left(t_{f M D}\right)-Z_{M D}(t)\right)^{2}\left[\int_{t}^{t_{f M T}} \boldsymbol{\alpha}^{2}(\tau) \mathrm{d} \tau+1 / a\right]}{2\left[\int_{t}^{t_{f M D}} \boldsymbol{\alpha}(\tau) \boldsymbol{\beta}(\tau) \mathrm{d} \tau\right]^{2}} .
$$

Then the following work focuses on the optimal choice of the variable $Z_{M D}\left(t_{f M D}\right)$ under the inequality constraint (28). Obviously, the optimal choice of $Z_{M D}\left(t_{f M D}\right)$ depends on the variable $Z_{M D}(t)$

$$
\begin{gathered}
Z_{M D}^{*}\left(t_{f M D}\right)= \\
\left\{\begin{array}{l}
Z_{M D}(t), \quad\left|Z_{M D}(t)\right| \geqslant M_{M D} \\
M_{M D} \cdot \operatorname{sign}\left(Z_{M D}(t)\right), \quad\left|Z_{M D}(t)\right|<M_{M D}
\end{array}\right.
\end{gathered}
$$

where $\operatorname{sign}(\cdot)$ is the signal function. Thus the optimal control can be derived from the inequality constraint point of view:

$$
u_{M}^{*}=\left\{\begin{array}{l}
0,\left|Z_{M D}(t)\right| \geqslant M_{M D} \\
\frac{\left[M_{M D} \operatorname{sign}\left(Z_{M D}(t)\right)-Z_{M D}(t)\right] \boldsymbol{\alpha}(t)}{\int_{t}^{t_{f M D}} \boldsymbol{\alpha}(\tau) \boldsymbol{\beta}(\tau) \mathrm{d} \tau}, \\
\left|Z_{M D}(t)\right|<M_{M D} .
\end{array}\right.
$$

Consequently, the optimal control $\boldsymbol{u}_{M}^{*}$ for the time duration $t_{0} \leqslant t \leqslant t_{f M D}$ is obtained from (51) and (59) under different conditions. For the time duration $t_{f M D}<t \leqslant$ $t_{f M T}$, the optimal control is given by (51). 
However, it should be declared that (59) is derived from the inequality constraint (28) rather than the performance index (42).

Remark 1 By detailed inspection, the optimal control $\boldsymbol{u}_{M}^{*}$ will keep zero if the condition given by (54) does not satisfy $\left|Z_{M D}(t)\right| \geqslant M_{M D}$, which implies that the optimal control $\boldsymbol{u}_{M}^{*}$ is considered as the optimal choice for the inequality constraint (28), but not an optimal choice for the performance index (42). In the time duration $t \in$ $\left[t_{0}, t_{f M D}\right]$, the control $\boldsymbol{u}_{M}^{*}$ keeps zero and does not do any contributions to the final miss $Z_{M T}\left(t_{f M T}\right)$, which, however, is regarded as the more significant part of the performance index.

Consequently, an additional part $\eta(t)$ is introduced to (51) to obtain a compromise between the inequality constraint (28) and the performance index (42), and thus the combined control can be expressed as follows:

$$
\boldsymbol{u}_{M}^{*}=\frac{-\eta(t) Z_{M T}(t) \boldsymbol{\alpha}(t)}{1 / a+\int_{t}^{t_{f M T}} \boldsymbol{\alpha}^{2}(\tau) \mathrm{d} \tau}
$$

with

$$
0 \leqslant \eta(t) \leqslant 1 .
$$

As a result, a compromise will be acquired if (60) is applied and the following equation is satisfied:

$$
\begin{gathered}
Z_{M D}(t)+\int_{t}^{t_{f M D}} \boldsymbol{\beta}(\tau) \frac{-\eta(\tau) Z_{M D}(t) \boldsymbol{\alpha}(\tau)}{1 / a+\int_{t}^{t_{f M D}} \boldsymbol{\alpha}^{2}(\kappa) \mathrm{d} \kappa} \mathrm{d} \tau= \\
M_{M D} \operatorname{sign}\left(Z_{M D}(t)\right) .
\end{gathered}
$$

Then, the following discussions on the choice of $\eta(t)$ can be conducted:

(i) $\eta(t) \equiv C$

In this case, the additional part $\eta(t)$ is chosen as a constant number. According to (62), the constant number will be

$$
\begin{gathered}
\eta(t) \equiv \\
\frac{\left[Z_{M D}(t)-M_{M D} \operatorname{sign}\left(Z_{M D}(t)\right)\right]\left[1 / a+\int_{t}^{t_{f M T}} \boldsymbol{\alpha}^{2}(\tau) \mathrm{d} \tau\right]}{Z_{M T}(t) \int_{t}^{t_{f M D}} \boldsymbol{\alpha}(\tau) \boldsymbol{\beta}(\tau) \mathrm{d} \tau} .
\end{gathered}
$$

Although the energy consumption increases in the time duration $\left[t_{0}, t_{f M D}\right]$, the final miss $Z_{M T}\left(t_{f M T}\right)$ is minimized to some extent. Meanwhile, the inequality constraint is constantly satisfied.

(ii) $\eta(t)=k_{1} t+k_{2}$

In this case, the additional part $\eta(t)$ is chosen as a linear function with regarding to time, and there are two parame- ters to be determined. Firstly, according to (62), the relation between the two parameters $\left(k_{1}, k_{2}\right)$ can be expressed as follows:

$$
k_{2}=A_{1} k_{1}+A_{2}
$$

where

$$
\begin{gathered}
A_{1}=-\frac{\int_{t}^{t_{f M T}} \boldsymbol{\alpha}(\tau) \boldsymbol{\beta}(\tau) \tau \mathrm{d} \tau}{\int_{t}^{t_{f} M T} \boldsymbol{\alpha}(\tau) \boldsymbol{\beta}(\tau) \mathrm{d} \tau} \\
\frac{\left[Z_{M D}(t)-M_{M D} \operatorname{sign}\left(Z_{M D}(t)\right)\right]\left[1 / a+\int_{t}^{t_{f M T}} \boldsymbol{\alpha}^{2}(\tau) \mathrm{d} \tau\right]}{Z_{M T}(t) \int_{t}^{t_{f M D}} \boldsymbol{\alpha}(\tau) \boldsymbol{\beta}(\tau) \mathrm{d} \tau} .
\end{gathered}
$$

Then substituting (60) into the performance index (42), it can be obtained that

$$
J=P \cdot f\left(k_{1}, k_{2}\right)
$$

where

$$
\begin{gathered}
P=\frac{a Z_{M T}(t)}{2\left[1+a \int_{t}^{t_{f M T}} \alpha^{2}(\tau) \mathrm{d} \tau\right]^{2}} \\
f\left(k_{1}, k_{2}\right)=B_{1} k_{1}^{2}+B_{2} k_{1} k_{2}+B_{3} k_{2}^{2}+B_{4} k_{1}+B_{5} k_{2}+B_{6}
\end{gathered}
$$

and

$$
\left\{\begin{aligned}
B_{1}= & a \int_{t}^{t_{f M T}} \boldsymbol{\alpha}^{2}(\tau) \tau^{2} \mathrm{~d} \tau+a^{2}\left[\int_{t}^{t_{f M T}} \boldsymbol{\alpha}^{2}(\tau) \tau \mathrm{d} \tau\right]^{2} \\
B_{2}= & 2 a \int_{t}^{t_{f M T}} \boldsymbol{\alpha}^{2}(\tau) \tau \mathrm{d} \tau+ \\
& 2 a^{2} \int_{t}^{t_{f} M T} \boldsymbol{\alpha}^{2}(\tau) \tau \mathrm{d} \tau \cdot \int_{t}^{t_{f M T}} \boldsymbol{\alpha}^{2}(\tau) \mathrm{d} \tau \\
B_{3}= & a \int_{t}^{t_{f M T}} \boldsymbol{\alpha}^{2}(\tau) \mathrm{d} \tau+a^{2}\left[\int_{t}^{t_{f M T}} \boldsymbol{\alpha}^{2}(\tau) \mathrm{d} \tau\right]^{2} \\
B_{4}= & -2 a \int_{t}^{t_{f M T}} \boldsymbol{\alpha}^{2}(\tau) \tau \mathrm{d} \tau- \\
& 2 a^{2} \int_{t}^{t_{f M T}} \boldsymbol{\alpha}^{2}(\tau) \tau \mathrm{d} \tau \cdot \int_{t}^{t_{f M T}} \boldsymbol{\alpha}^{2}(\tau) \mathrm{d} \tau \\
B_{5}= & -2 a \int_{t}^{t_{f M T}} \boldsymbol{\alpha}^{2}(\tau) \mathrm{d} \tau-2 a^{2}\left[\int_{t}^{t_{f M T}} \boldsymbol{\alpha}^{2}(\tau) \mathrm{d} \tau\right]^{2} \\
B_{6}= & {\left[1+a \int_{t}^{t_{f M T}} \alpha^{2}(\tau) \mathrm{d} \tau\right]^{2} }
\end{aligned}\right.
$$

Besides, combining with (64), the performance index can be transformed into the following expression:

$$
J=P \cdot\left(C_{1} k_{1}^{2}+C_{2} k_{1}+C_{3}\right)
$$


where

$$
\left\{\begin{array}{l}
C_{1}=B_{1}+B_{2} A_{1}+B_{3} A_{1}^{2} \\
C_{2}=B_{2} A_{2}+2 B_{3} A_{1} A_{2}+B_{4}+B_{5} A_{1} . \\
C_{3}=B_{3} A_{2}^{2}+B_{5} A_{2}+B_{6}
\end{array} .\right.
$$

Here a new parameter is defined as

$$
k_{1}^{*} \triangleq \frac{-C_{2}}{2 C_{1}} .
$$

Due to the constraint (61), the scope of $k_{1}$ is determined, which is marked as follows:

$$
k_{1} \in\left[k_{1 l}, k_{1 h}\right] .
$$

Thus the optimal choice of $k_{1}$ will be

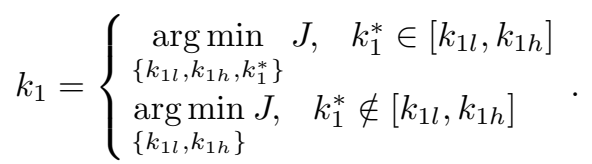

And the optimal ohoice of $k_{2}$ is determined by (64).

Notice that if the additional part $\eta(t)$ is chosen as a second or high order function of time, all the parameters can not be determined absolutely without additional relations or conditions.

On the whole, the optimal control $\boldsymbol{u}_{M}^{*}$ for the time duration $t \in\left[t_{0}, t_{f M D}\right]$ can be obtained by a compromise technique, which is given by (76). In addition, for the time duration $t \in\left[t_{f M D}, t_{f M T}\right]$, the optimal control $\boldsymbol{u}_{M}^{*}$ is given by $(51)$.

$$
\begin{aligned}
& \boldsymbol{u}_{M}^{*}=\left\{\begin{array}{l}
\frac{-Z_{M T}(t) \boldsymbol{\alpha}(t)}{1 / a+\int_{t}^{t_{f M T}} \boldsymbol{\alpha}^{2}(\tau) \mathrm{d} \tau}, \quad(54) \text { is satisfied } \\
\frac{-\eta(t) Z_{M T}(t) \boldsymbol{\alpha}(t)}{1 / a+\int_{t}^{t_{f M T}} \boldsymbol{\alpha}^{2}(\tau) \mathrm{d} \tau}, \quad(54) \text { is not } \\
\text { satisfied and }\left|Z_{M D}(t)\right| \geqslant M_{M D} \\
\frac{\left[M_{M D} \operatorname{sign}\left(Z_{M D}(t)\right)-Z_{M D}(t)\right] \boldsymbol{\alpha}(t)}{\int_{t}^{t_{f M D}} \boldsymbol{\alpha}(\tau) \boldsymbol{\beta}(\tau) \mathrm{d} \tau}
\end{array}\right. \\
& \text { (54) is not satisfied and } \\
& \left|Z_{M D}(t)\right|<M_{M D}
\end{aligned}
$$

\section{Guidance algorithm with control saturations}

In the last section, the optimal control for the linearized model is derived without control saturations. Nevertheless, it has been found that in most practical situations, there exist the following phenomena:

(i) The absolute values of $Z_{M D}(t)$ and $Z_{M D}\left(t_{f M D}\right)$ approach to zero and are pretty smaller than $M_{M D}$.

(ii) On the basis of (i), the homing missile must evade the defender first and then intercept the target. However, the optimal control $\boldsymbol{u}_{M}^{*}$ calculated by (76) will be too large to be implemented practically.

\subsection{Introduction to nonlinear saturation factors}

With the above analysis, the control saturations should be introduced to getting access to a more reasonable view of the integral TMD problem. When saturations occur, the structure of the system equations varies and the dynamics of zero-effort miss distances become different from the original process.

Under the assumed cooperative strategies of the targetdefender team, when the defender's control $\boldsymbol{u}_{D}$ saturates at time $t_{D s}$, the state equation becomes

$$
\begin{gathered}
\dot{\boldsymbol{x}}=\boldsymbol{A} \boldsymbol{x}+\boldsymbol{B}_{D} \boldsymbol{u}_{D \max } \operatorname{sign}\left(Z_{M D}(t)\right)+ \\
\boldsymbol{B}_{T} \boldsymbol{u}_{T}(t)+\boldsymbol{B}_{M} \boldsymbol{u}_{M}(t)
\end{gathered}
$$

where $\boldsymbol{u}_{D \text { max }}$ denotes the maximum acceleration command of the defender, which leads to new state transition matrices $\boldsymbol{\Phi}_{T D}^{\prime}\left(t_{f M T}, t\right)$ and $\boldsymbol{\Phi}_{T D}^{\prime}\left(t_{f M D}, t\right)$. Consequently, in different time durations, the calculation of $\alpha(t)$ and $\beta(t)$ depends on whether saturations occur or not.

To make the proposed guidance algorithm readily applicable for practical situations, the following case is considered that all three agents have first-order dynamics, which are expressed as follows:

$A_{i}=-\frac{1}{\tau_{i}}, \quad b_{i}=\frac{1}{\tau_{i}}, \quad c_{i}=1, \quad d_{i}=0, \quad i=\{T, M, D\}$.

It is defined that

$$
\left\{\begin{array}{l}
\boldsymbol{X}_{M T}\left(t_{g o M T}\right)=\boldsymbol{\Phi}_{T D}\left(t_{f M T}, t\right) \boldsymbol{d}_{M T}^{\mathrm{T}} \\
\boldsymbol{X}_{M D}\left(t_{g o M D}\right)=\boldsymbol{\Phi}_{T D}\left(t_{f M D}, t\right) \boldsymbol{d}_{M D}^{\mathrm{T}}
\end{array}\right.
$$

then

$$
\left\{\begin{array}{l}
\dot{\boldsymbol{X}}_{i}\left(t_{g o i}\right)=\boldsymbol{A}_{T D}^{\mathrm{T}}\left(t_{f i}-t\right) \boldsymbol{X}_{i}\left(t_{g \circ i}\right), \quad i \in\{M T, M D\} . \\
\boldsymbol{X}_{i}(0)=d_{i}
\end{array}\right.
$$

Therefore, the state-space vector will be

$$
\boldsymbol{x}=\left[y_{M T}, \dot{y}_{M T}, a_{T}, y_{M D}, \dot{y}_{M D}, a_{D}, a_{M}\right]^{\mathrm{T}} .
$$

Obviously, the state matrix $\boldsymbol{A}_{T D}$ has different forms with regarding to the case whether the defender's acceleration command is saturated. As a result, the dynamics of zero-effort miss distances vary as well.

Remark 2 If the acceleration command does not saturates, the state matrix $\boldsymbol{A}_{I D}$ and the variation of zero-effort miss distances will be the same with that of the linear case. In this case, the defender's acceleration command is produced according to the current state-space vector, while the dynamics of the zero-effort miss distances still have nothing to do with the defender's acceleration command. The state matrix is given by (82). Besides, (40) holds for the zero-effort miss distances. However, if it saturates, the state matrix $\boldsymbol{A}_{T D}^{\prime}$ will be expressed by (84), and the zero-effort $\operatorname{miss} Z_{M D}^{\prime}(t)$ satisfies (85). 


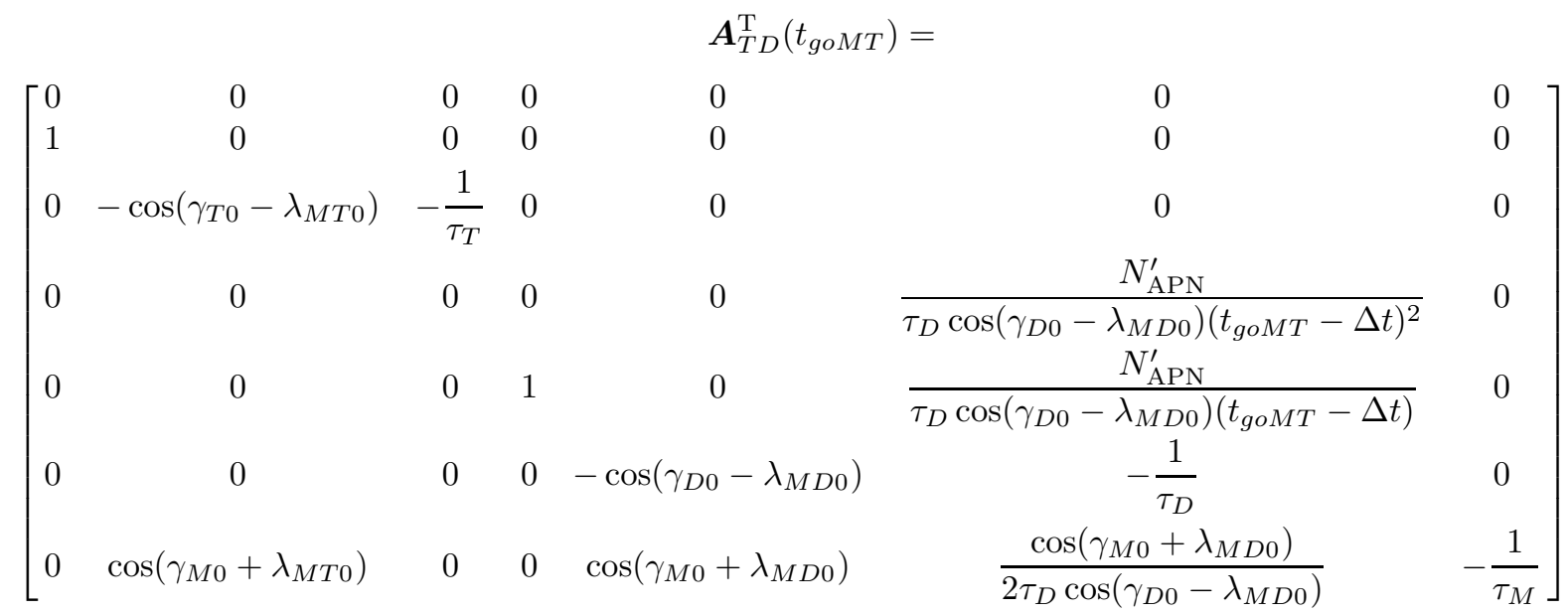

where

$$
\Delta t=t_{f M T}-t_{f M D}
$$

$$
\boldsymbol{A}_{T D}^{\prime \mathrm{T}}\left(t_{g o M T}\right)=\left[\begin{array}{ccccccc}
0 & 0 & 0 & 0 & 0 & 0 & 0 \\
1 & 0 & 0 & 0 & 0 & 0 & 0 \\
0 & -\cos \left(\gamma_{T 0}-\lambda_{M T 0}\right) & -\frac{1}{\tau_{T}} & 0 & 0 & 0 & 0 \\
0 & 0 & 0 & 0 & 0 & 0 & 0 \\
0 & 0 & 0 & 1 & 0 & 0 & 0 \\
0 & 0 & 0 & 0 & -\cos \left(\gamma_{D 0}-\lambda_{M D 0}\right) & -\frac{1}{\tau_{D}} & 0 \\
0 & \cos \left(\gamma_{M 0}+\lambda_{M T 0}\right) & 0 & 0 & \cos \left(\gamma_{M 0}+\lambda_{M D 0}\right) & 0 & -\frac{1}{\tau_{M}}
\end{array}\right]
$$

$$
\dot{Z}_{M D}^{\prime}(t)=\left\{\begin{array}{c}
\beta(t) \boldsymbol{u}_{M}, \quad 0 \leqslant t \leqslant t_{D s} \\
\beta(t) \boldsymbol{u}_{M}+X_{M D}^{\mathrm{T}}\left(t_{g o M D}\right) B_{D} \boldsymbol{u}_{D} \\
t_{D s} \leqslant t \leqslant t_{f M D}
\end{array}\right.
$$

where

$$
\boldsymbol{u}_{D}=\boldsymbol{u}_{D \max } \operatorname{sign}\left(Z_{M D}^{\prime}\left(t_{0}\right)\right) \text {. }
$$

\subsection{Solution to the original linear optimal problem}

For the implementation of the guidance algorithm in an efficiency manner, it is inspiring to make full use of adjoint solutions. The vectors $\boldsymbol{X}_{M T}\left(t_{g o M T}\right)$ and $\boldsymbol{X}_{M D}\left(t_{g o M D}\right)$ can be expressed in detail, which are given as follows:

$$
\left\{\begin{array}{l}
\boldsymbol{X}_{M T}\left(t_{g o M T}\right)=\left[\varepsilon_{1}\left(t_{g o M T}\right), \ldots, \varepsilon_{7}\left(t_{g o M T}\right)\right]^{\mathrm{T}} \\
\boldsymbol{X}_{M D}\left(t_{g o M D}\right)=\left[\sigma_{1}\left(t_{g o M D}\right), \ldots, \sigma_{7}\left(t_{g o M D}\right)\right]^{\mathrm{T}}
\end{array}\right.
$$

with the initial condition

$$
\left\{\begin{array} { l } 
{ \varepsilon _ { 1 } ( 0 ) = 1 } \\
{ \varepsilon _ { i } ( 0 ) = 0 , i \neq 1 }
\end{array} \text { and } \left\{\begin{array}{l}
\sigma_{4}(0)=1 \\
\sigma_{i}(0)=0, i \neq 4
\end{array} .\right.\right.
$$

For the time duration $t \in\left(t_{f M D}, t_{f M T}\right]$, that is, $t_{g o M T} \in[0, \Delta t)$, the analytical solution of $\boldsymbol{X}_{M T}\left(t_{g o M T}\right)$ can be derived, which is given by

$$
\left\{\begin{array}{c}
\varepsilon_{1}\left(t_{g \circ M T}\right)=1 \\
\varepsilon_{2}\left(t_{g o M T}\right)=t_{g o M T} \\
\varepsilon_{3}\left(t_{g \circ M T}\right)=\cos \left(\gamma_{T 0}-\lambda_{M T 0}\right) \tau_{T}^{2} . \\
\quad\left(1-\frac{t_{g o M T}}{\tau_{T}}-\mathrm{e}^{-t_{g o M T} / \tau_{T}}\right) \\
\varepsilon_{4}\left(t_{g o M T}\right)=\varepsilon_{5}\left(t_{g o M T}\right)=\varepsilon_{6}\left(t_{g o M T}\right)=0 \\
\varepsilon_{7}\left(t_{g o M T}\right)=-\cos \left(\gamma_{M 0}+\lambda_{M T 0}\right) \tau_{M}^{2} . \\
\quad\left(1-\frac{t_{g o M T}}{\tau_{M}}-\mathrm{e}^{-t_{g \circ M T} / \tau_{M}}\right)
\end{array} .\right.
$$

For the time duration $t \in\left[0, t_{f M D}\right]$, that is, $t_{g o M T} \in$ $\left[\Delta t, t_{f M T}\right]$ and $t_{g o M D} \in\left[0, t_{f M D}\right]$, the analytical solution of $\boldsymbol{X}_{M T}\left(t_{g o M T}\right)$ can be derived, given by (93), where

$$
\left\{\begin{array}{l}
g_{1}(\theta, \omega)=-\frac{1}{2}\left(\frac{\theta^{2}}{2}-\frac{\theta^{3}}{6}\right) \\
g_{2}(\theta, \omega)=\left(\theta-\frac{\theta^{2}}{2}\right)\left(\omega-\frac{1}{2}\right) \\
g_{3}(\theta, \omega)=-(1-\theta)\left(\omega-\frac{1}{2}\right) \\
g_{4}(\theta, \omega)=\left(\omega-\frac{1}{2}\right)
\end{array} .\right.
$$


The analytical solution of $\boldsymbol{X}_{M D}\left(t_{g o M D}\right)$ can be derived similarly, given by (94). Thus, the analytical solution of the optimal problem is obtained.

\subsection{Solution to the optimal problem with nonlinear saturation factor}

For the time duration $t \in\left(t_{f M D}, t_{f M T}\right]$, the analytical solution of $\boldsymbol{X}_{M T}\left(t_{g o M T}\right)$ is the same as that of the linear optimal problem.

For the time duration $t \in\left(t_{D s}, t_{f M D}\right]$, that is, $t_{g o M T} \in$ $\left[\Delta t, t_{f M T}-t_{D s}\right)$, the analytical solution of $\boldsymbol{X}_{M T}\left(t_{g o M T}\right)$ can be given by (91).

Similarly, the corresponding duration for $t_{g o M D}$ will be $t_{g o M D} \in\left[0, t_{f M D}-t_{D s}\right)$. Thus the analytical solution of $\boldsymbol{X}_{M D}\left(t_{\text {goMD }}\right)$ can be expressed by (92).

For the time duration $t \in\left[0, t_{D s}\right]$, that is, $t_{g o M T} \in$ $\left[t_{f M T}-t_{D s}, t_{f M T}\right]$ and $t_{g o M D} \in\left[t_{f M D}-t_{D s}, t_{f M D}\right]$, the dynamics of the vectors $\boldsymbol{X}_{M T}\left(t_{g o M T}\right)$ and $\boldsymbol{X}_{M D}\left(t_{g o M D}\right)$ can be determined by (80). The state-space matrix $A_{T D}$ is given by (82). Unfortunately, the abrupt variation of the state-space matrix $\boldsymbol{A}_{T D}$ results in the discontinuity of the elements' differentials of the two vectors. Thus, the analytical solution cannot be obtained. As a result, the solutions of the vectors $\boldsymbol{X}_{M T}\left(t_{g o M T}\right)$ and $\boldsymbol{X}_{M D}\left(t_{g o M D}\right)$ will be acquired by numerical methods and the fourth-order Runge-Kutta algorithm is applied.

$$
\begin{aligned}
& \left\{\begin{array}{l}
\varepsilon_{1}\left(t_{g o M T}\right)=\varepsilon_{4}\left(t_{g o M T}\right)=1 \\
\varepsilon_{2}\left(t_{g o M T}\right)=t_{g o M T} \\
\varepsilon_{3}\left(t_{g o M T}\right)=\cos \left(\gamma_{T 0}-\lambda_{M T 0}\right) \tau_{T}^{2}\left(1-\frac{t_{g o M T}}{\tau_{T}}-\mathrm{e}^{-t_{g o M T} / \tau_{T}}\right) \\
\varepsilon_{5}\left(t_{g o M T}\right)=t_{g o M T}-\Delta t \\
\varepsilon_{6}\left(t_{g o M T}\right)=\cos \left(\gamma_{D 0}-\lambda_{M D 0}\right) \tau_{D}^{2}\left(1-\frac{t_{g o M T}-\Delta t}{\tau_{D}}-\mathrm{e}^{-\left(t_{g o M T}-\Delta t\right) / \tau_{D}}\right) \\
\varepsilon_{7}\left(t_{g o M T}\right)=-\cos \left(\gamma_{M 0}+\lambda_{M T 0}\right) \tau_{M}^{2}\left(1-\frac{t_{g o M T}}{\tau_{M}}-\mathrm{e}^{-t_{g o M T} / \tau_{M}}\right) \\
\quad-\cos \left(\gamma_{M 0}+\lambda_{M D 0}\right) \tau_{D}^{2}\left(1-\frac{t_{g o M T}-\Delta t}{\tau_{D}}-\mathrm{e}^{-\left(t_{g o M T}-\Delta t\right) / \tau_{D}}\right)
\end{array}\right. \\
& \left\{\begin{array}{l}
\sigma_{1}\left(t_{g o M D}\right)=\sigma_{2}\left(t_{g o M D}\right)=\sigma_{3}\left(t_{g o M D}\right)=0 \\
\sigma_{4}\left(t_{g o M D}\right)=1 \\
\sigma_{5}\left(t_{g o M D}\right)=t_{g o M D} \\
\sigma_{6}\left(t_{g o M D}\right)=\cos \left(\gamma_{D 0}-\lambda_{M D 0}\right) \tau_{D}^{2}\left(1-\frac{t_{g o M D}}{\tau_{D}}-\mathrm{e}^{-\frac{t_{g o M D}}{\tau_{D}}}\right) \\
\sigma_{7}\left(t_{g o M D}\right)=-\cos \left(\gamma_{M 0}+\lambda_{M D 0}\right) \tau_{M}^{2}\left(1-\frac{t_{g o M D}}{\tau_{M}}-\mathrm{e}^{-\frac{t_{g o M D}}{\tau_{M}}}\right)
\end{array}\right.
\end{aligned}
$$

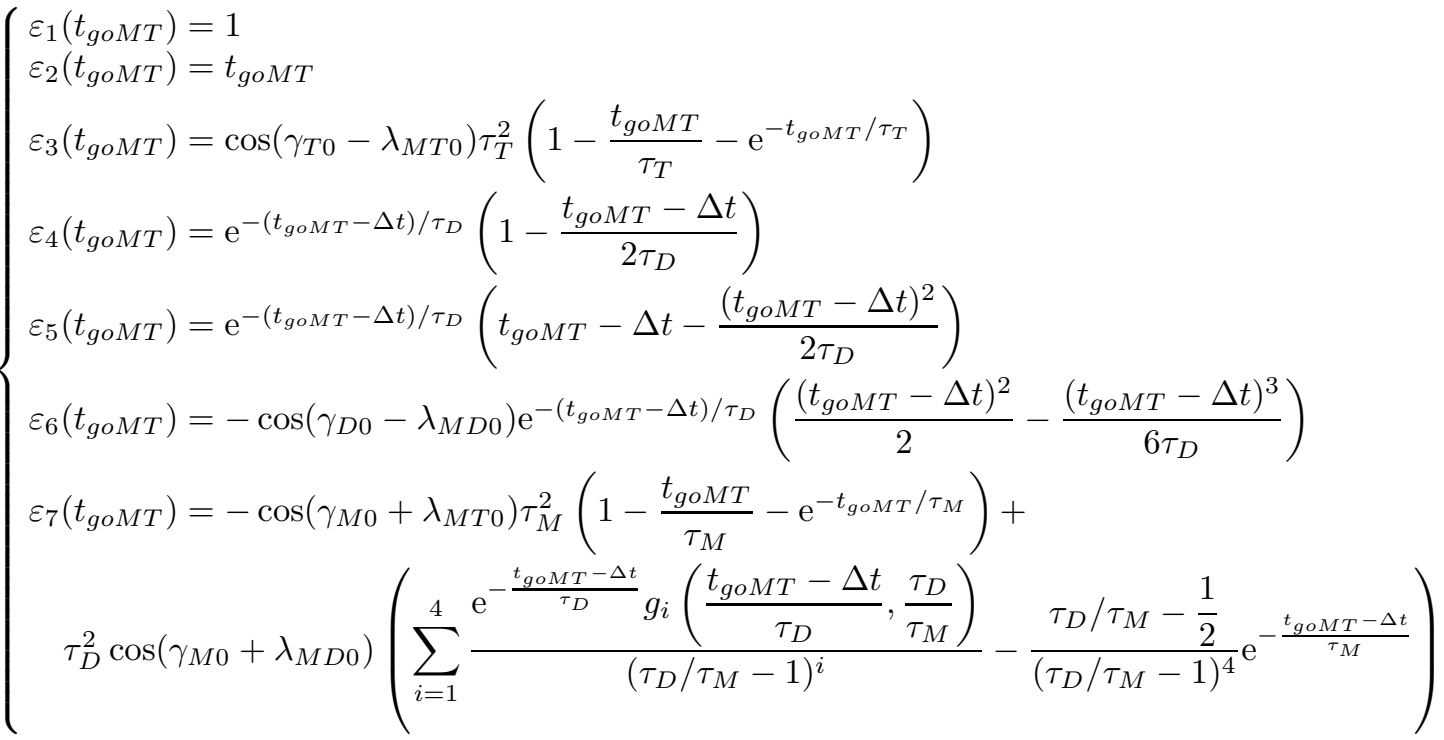




$$
\left\{\begin{array}{l}
\sigma_{1}\left(t_{g o M D}\right)=\sigma_{2}\left(t_{g o M D}\right)=\sigma_{3}\left(t_{g o M D}\right)=0 \\
\sigma_{4}\left(t_{g o M D}\right)=\left(1-\frac{t_{g o M D}}{2 \tau_{D}}\right) \mathrm{e}^{-t_{g o M D} / \tau_{D}} \\
\sigma_{5}\left(t_{g o M D}\right)=t_{g o M D}\left(1-\frac{t_{g o M D}}{2 \tau_{D}}\right) \mathrm{e}^{-t_{g o M D} / \tau_{D}} \\
\sigma_{6}\left(t_{g o M D}\right)=-\cos \left(\gamma_{D 0}-\lambda_{M D 0}\right) t_{g o M D}^{2}\left(\frac{1}{2}-\frac{t_{g o M D}}{6 \tau_{D}}\right) \mathrm{e}^{-t_{g o M D} / \tau_{D}} \\
\sigma_{7}\left(t_{g o M D}\right)=\tau_{D}^{2} \cos \left(\gamma_{M 0}+\lambda_{M D 0}\right)\left(\sum_{i=1}^{4} \frac{\mathrm{e}^{-\frac{t_{g o M D}}{\tau_{D}}} g_{i}\left(\frac{t_{g o M D}}{\tau_{D}}, \frac{\tau_{D}}{\tau_{M}}\right)}{\left(\tau_{D} / \tau_{M}-1\right)^{i}}-\frac{1}{\tau_{D} / \tau_{M}-\frac{1}{2}} \frac{\left.\tau_{D} / \tau_{M}-1\right)^{4}}{-\frac{t_{g o M D}}{\tau_{M}}}\right)
\end{array}\right.
$$

Once $\boldsymbol{X}_{M T}\left(t_{g o M T}\right)$ and $\boldsymbol{X}_{M D}\left(t_{g o M D}\right)$ are obtained, the vectors $\boldsymbol{\alpha}(t)$ and $\boldsymbol{\beta}(t)$ will be calculated by (40) and (79). Next, the zero-effort miss distances $Z_{M T}(t)$ and $Z_{M D}(t)$ can be acquired by (37) and (38) respectively. Eventually, the optimal control $\boldsymbol{u}_{M}^{*}$ will be given by (76).

\subsection{Iterative technique for the determination of saturation time points}

The aforementioned methods in Section 4 keep silent to the determination of the saturation time points for the defender and the missile. This two-point boundary value problem with nonlinear saturation factor is difficult to solve directly or indirectly. Therefore, an efficient iterative technique is proposed to determine the saturation time point $t_{D s}$ for the defender and $t_{M s}$ for the missile, which is illustrated in Fig. 2.

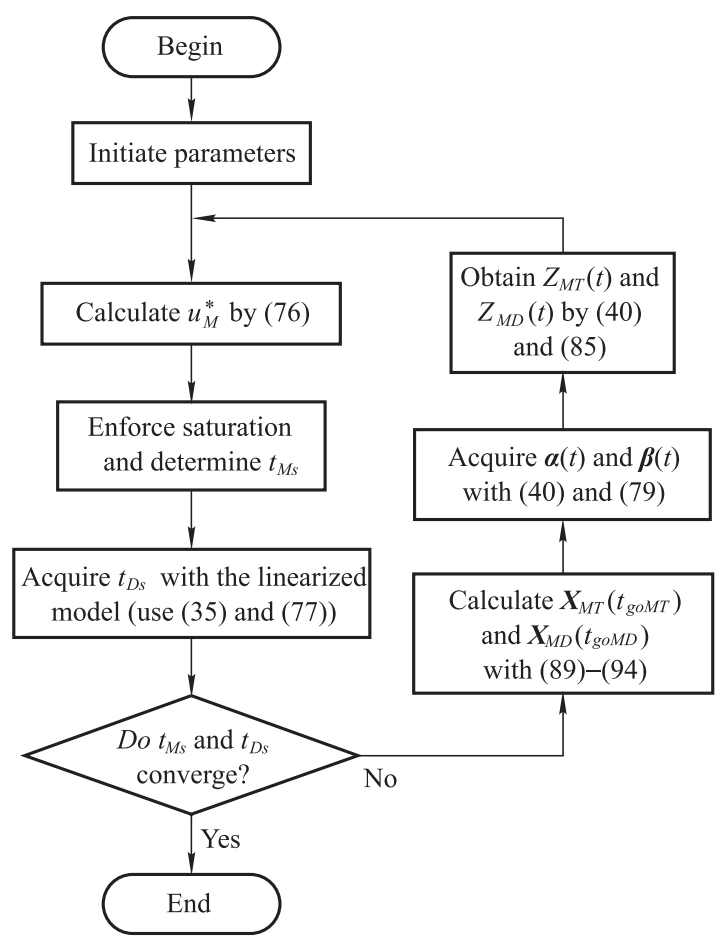

Fig. 2 Flow chart of iterative algorithm

\section{Simulation results}

To get further insight in the effectiveness of the proposed algorithm, the results of the numerical simulation are presented.

\subsection{Linear optimal problem without control saturations}

With regarding to the linear optimal problem without control saturations, the measurements on the inequality constraint are evaluated. The relevant parameters are shown in Table 1.

Table 1 Parameter values for the performance simulation of the linear optimal problem

\begin{tabular}{cc}
\hline Parameter & Value \\
\hline$V_{T} /(\mathrm{m} / \mathrm{s})$ & 300 \\
$V_{D} /(\mathrm{m} / \mathrm{s})$ & 600 \\
$V_{M} /(\mathrm{m} / \mathrm{s})$ & 600 \\
$\tau_{T} / \mathrm{s}$ & 1.0 \\
$\tau_{D} / \mathrm{s}$ & 1.0 \\
$\tau_{M} / \mathrm{s}$ & 0.1 \\
$u_{T \text { max }} /\left(\mathrm{m} / \mathrm{s}^{2}\right)$ & 49 \\
$\left(x_{T 0}, y_{T 0}\right) / \mathrm{m}$ & $(0,0)$ \\
$\left(x_{D 0}, y_{D 0}\right) / \mathrm{m}$ & $(2000,0)$ \\
$\left(x_{M 0}, y_{M 0}\right) / \mathrm{m}$ & $(10000,500)$ \\
$\left(\gamma_{T 0}, \gamma_{D 0}, \gamma_{M 0}\right) /\left(^{\circ}\right)$ & $(0,0,0)$ \\
$N_{P N}^{\prime}$ & 1.3 \\
$M_{M D} / \mathrm{m}$ & 20 \\
Step length $h / \mathrm{s}$ & 0.001 \\
\hline
\end{tabular}

It should be emphasized that to evaluate the measurements tackling the inequality constraint (28), the control saturation is ignored temporarily. Meanwhile, the guidance law of the defender is assumed to be pure proportional navigation (PPN) guidance.

Remark 3 The defender's guidance law seems inadequate to some extent. However, in this case, the situation that the zero-effort miss $\left|Z_{M D}(t)\right| \geqslant M_{M D}$ will appear, then the proposed measurements can be validated sufficiently.

In addition, the vectors of $\boldsymbol{X}_{M T}\left(t_{g o M T}\right)$ and 
$\boldsymbol{X}_{M D}\left(t_{g o M D}\right)$ are obtained from numerical algorithms according to (80).

Fig. 3 and Fig. 4 present the vectors of $\boldsymbol{X}_{M T}\left(t_{g o M T}\right)$ and $\boldsymbol{X}_{M D}\left(t_{g o M D}\right)$ from the linearized model of (35). The elements $\varepsilon_{1}, \varepsilon_{2}, \varepsilon_{3}$ and $\sigma_{1}, \sigma_{2}, \sigma_{3}$ have been derived in (93) and (94) analytically without numerical methods. In Fig. 3, there are sharp variations at the time point $t_{f M D}$ because of the end of the process between the missile and the defender. Besides, the variations of $\varepsilon_{4}, \varepsilon_{5}, \varepsilon_{6}$ and $\sigma_{4}, \sigma_{5}, \sigma_{6}$ for the time duration $t \in\left[t_{0}, t_{f M D}\right]$ are correspondingly similar due to the identical dynamics. However, the variations of $\varepsilon_{7}$ and $\sigma_{7}$ are significantly discrepant because of their different descriptions of the engagement process.

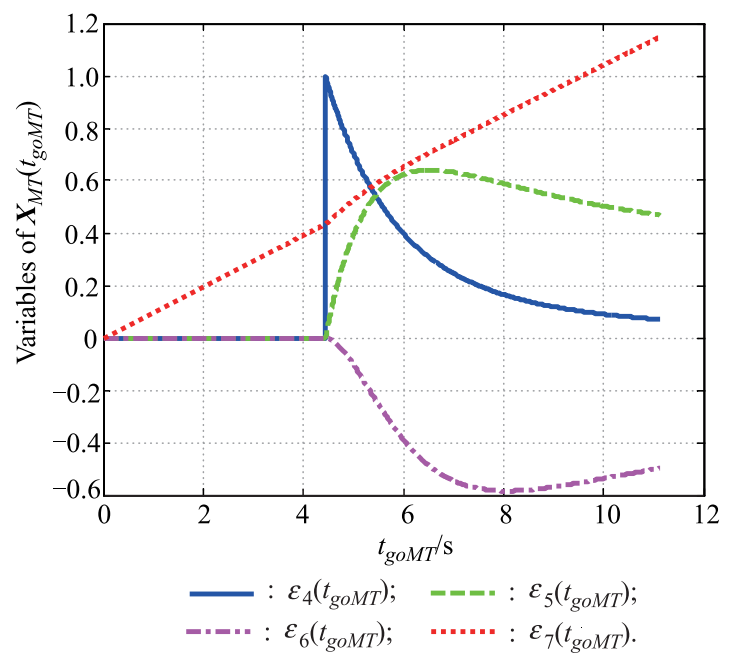

Fig. 3 Profiles of the vectors of $X_{M T}\left(t_{g o M T}\right)$

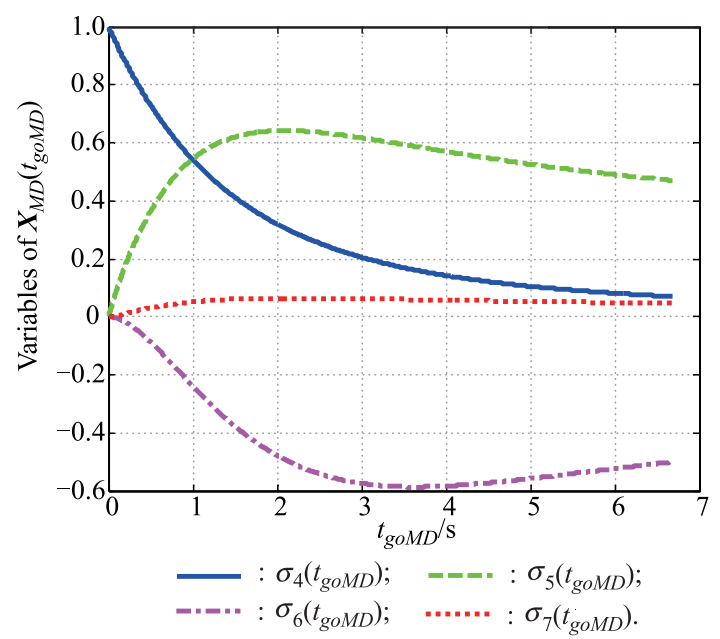

Fig. 4 Profiles of the vectors of $X_{M D}\left(t_{g o M D}\right)$

Fig. 5 and Fig. 6 illustrate the control profiles of the homing missile and the defender in the open loop form respectively. These plots demonstrate very clearly how the guidance commands change when the engagement of the defender is finished. There is a sharp variation in the missile's acceleration command at the terminal time $t_{f M D}$ to decrease the zero-effort miss $Z_{M T}(t)$, which is originally enlarged due to the missile's evasion from the defender. The defender's acceleration command diverges along with its approaching to the missile.

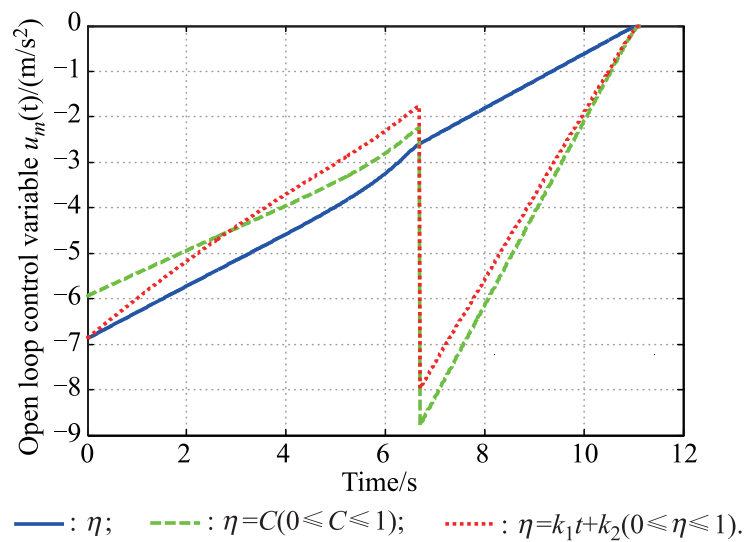

Fig. 5 Control profiles of the homing missile in open loop form of the linearized model

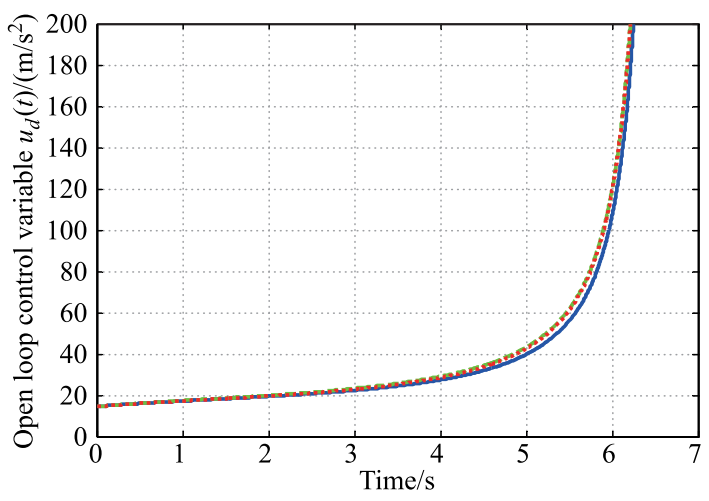

- $\eta ; \quad--=: \eta=C(0 \leqslant C \leqslant 1) ; \quad \cdots \cdots: \cdots: \eta=k_{1} t+k_{2}(0 \leqslant \eta \leqslant 1)$.

Fig. 6 Control profiles of the defender in open loop form of the linearized model

Fig. 7 and Fig. 8 demonstrate the profiles of the zeroeffort miss distances $Z_{M T}(t)$ and $Z_{M D}(t)$, respectively.

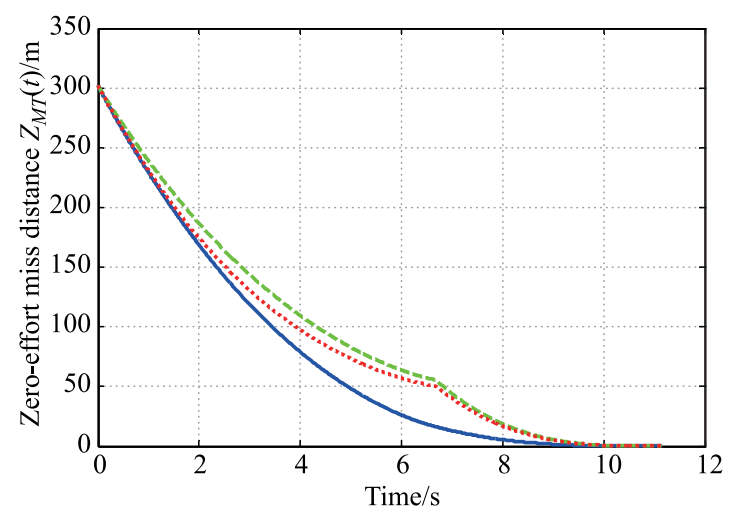

- $\eta ; \quad---:: \eta=C(0 \leqslant C \leqslant 1) ; \quad \cdots \cdots \cdot \cdots: \eta=k_{1} t+k_{2}(0 \leqslant \eta \leqslant 1)$.

Fig. 7 Profiles of the zero-effort miss distance $Z_{M T}(t)$ of the linearized model 


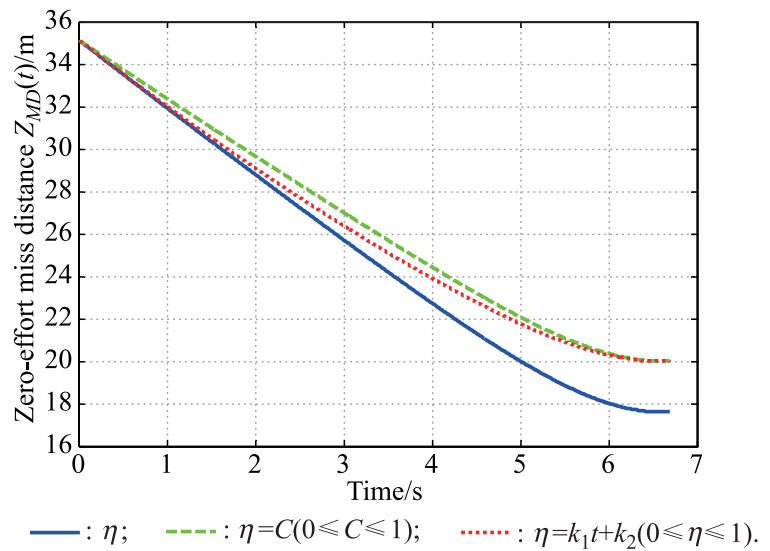

Fig. 8 Profiles of the zero-effort miss distance $Z_{M D}(t)$ of the linearized model

Obviously, the $Z_{M D}(t)$ guarantees the successful evasion from the defender with a specific distance. The evasion has some influence on the missile's interception for the target, due to the fact that the diminution of the variable $Z_{M T}(t)$ is delayed to some extent. However, a desirable value of $Z_{M T}\left(t_{f M T}\right)$ can be obtained for a successful interception for the target.

Fig. 9 illustrates a typical trajectory in the nonlinear kinematics. The corresponding closed loop control profiles are shown in Fig. 10. As appeared, the homing missile utilizes fewer efforts to evade the defender and hereafter intercepts the target perfectly. The introduction of $\eta(t)$ guarantees the successful evasion from the defender and minimizes the energy consumption to some extent.

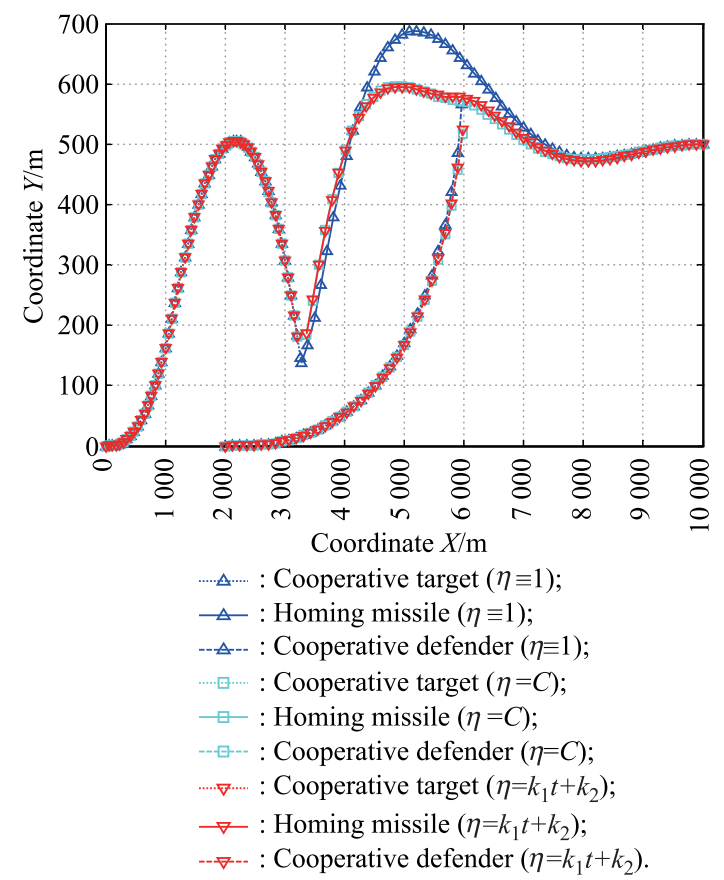

Fig. 9 Trajectory profiles for a homing missile using smart guidance law with nonlinear kinematics

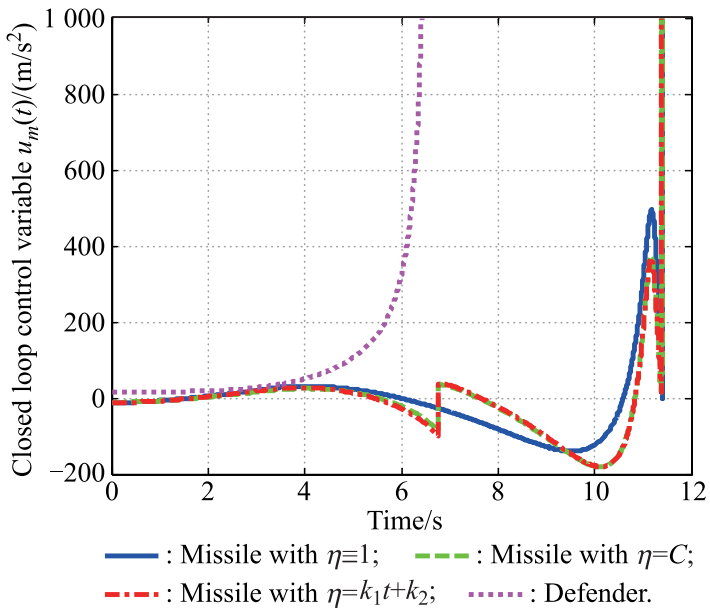

Fig. 10 Control profiles of the missile and defender in the closed loop form of the nonlinear kinematics

From Fig. 10, it is obvious that in the nonlinear kinematics, the closed loop control is much larger than that in the linear kinematics due to the error between the two kinds of kinematics. However, under both kinds of kinematics, the acceleration command of the homing missile is much smaller than that of the defender. This phenomenon further confirms the optimality of the proposed guidance algorithm in terms of the minimum energy consumption. In addition, the miss distance $y\left(t_{f M D}\right)$ is approximate to 60 $\mathrm{m}$, larger than the specific miss distance $M_{M D}$. Therefore, the linearization process of the integral TMD problem is conservative.

\subsection{Optimal problem with nonlinear saturation factor}

In this subsection, the performance of the smart guidance algorithm with nonlinear control saturations is investigated. The relevant parameters are listed in Table 2.

Table 2 Parameter values for the performance simulation of the optimal problem with nonlinear control saturations

\begin{tabular}{cc}
\hline Parameter & Value \\
\hline$V_{T} /(\mathrm{m} / \mathrm{s})$ & 300 \\
$V_{D} /(\mathrm{m} / \mathrm{s})$ & 600 \\
$V_{M} /(\mathrm{m} / \mathrm{s})$ & 600 \\
$\tau_{T} / \mathrm{s}$ & 1.0 \\
$\tau_{D} / \mathrm{s}$ & 0.1 \\
$\tau_{M} / \mathrm{s}$ & 0.1 \\
$u_{T \max } /\left(\mathrm{m} / \mathrm{s}^{2}\right)$ & 49 \\
$u_{D \max } /\left(\mathrm{m} / \mathrm{s}^{2}\right)$ & 147 \\
$u_{M \max } /\left(\mathrm{m} / \mathrm{s}^{2}\right)$ & 147 \\
$\left(x_{T 0}, y_{T 0}\right) / \mathrm{m}$ & $(0,0)$ \\
$\left(x_{D 0}, y_{D 0}\right) / \mathrm{m}$ & $(2000,0)$ \\
$\left(x_{M 0}, y_{M 0}\right) / \mathrm{m}$ & $(10000,500)$ \\
$\left(\gamma_{T 0}, \gamma_{D 0}, \gamma_{M 0}\right) /\left({ }^{\circ}\right)$ & $(0,0,0)$ \\
$N_{P N}^{\prime}$ & 3 \\
$\left(M_{M D}, M_{M T}\right) / \mathrm{m}$ & $(20,0)$ \\
Step Length $h / \mathrm{s}$ & 0.001 \\
$a$ & 1000 \\
\hline
\end{tabular}


Under the circumstance described in Table 2, the zeroeffort miss distance $Z_{M D}(t)$ is pretty small in the whole duration and the control saturations of three agents should be introduced to get reasonable insight on the TMD problem. With the iterative searching technique proposed in Section 4, the convergent results turn out that

$$
\left\{\begin{array}{l}
t_{D s}=3.617 \mathrm{~s} \\
t_{M s}=0
\end{array}\right.
$$

which means that the defender's acceleration command is saturated at time $t=3.617 \mathrm{~s}$ while the homing missile is not saturated during the whole process.

Fig. 11 and Fig. 12 present the profiles of the vectors of $\boldsymbol{X}_{M T}\left(t_{g o M T}\right)$ and $\boldsymbol{X}_{M D}\left(t_{g o M D}\right)$ without control saturations, respectively. Notice that the elements $\varepsilon_{1}, \varepsilon_{2}, \varepsilon_{3}$ and $\sigma_{1}, \sigma_{2}, \sigma_{3}$ have analytical solutions according to (93) and (94) respectively.

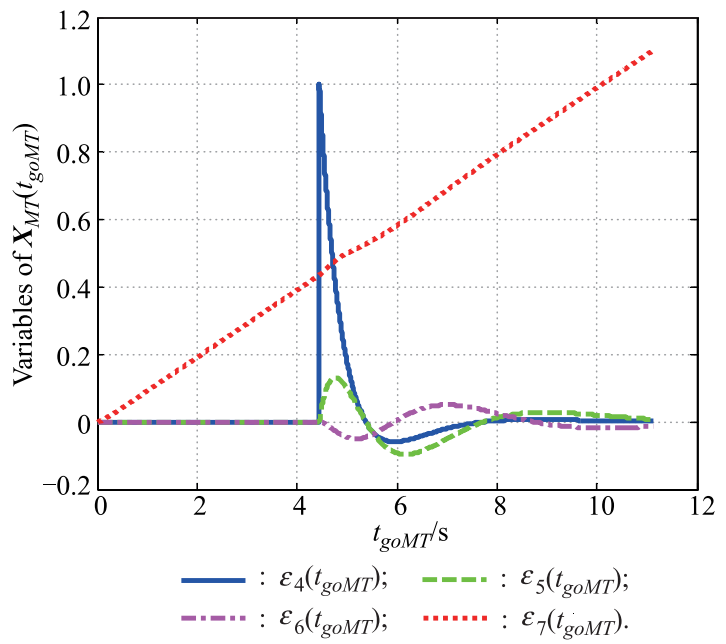

Fig. 11 Profiles of the vectors of $X_{M T}\left(t_{g o M T}\right)$ without control saturations

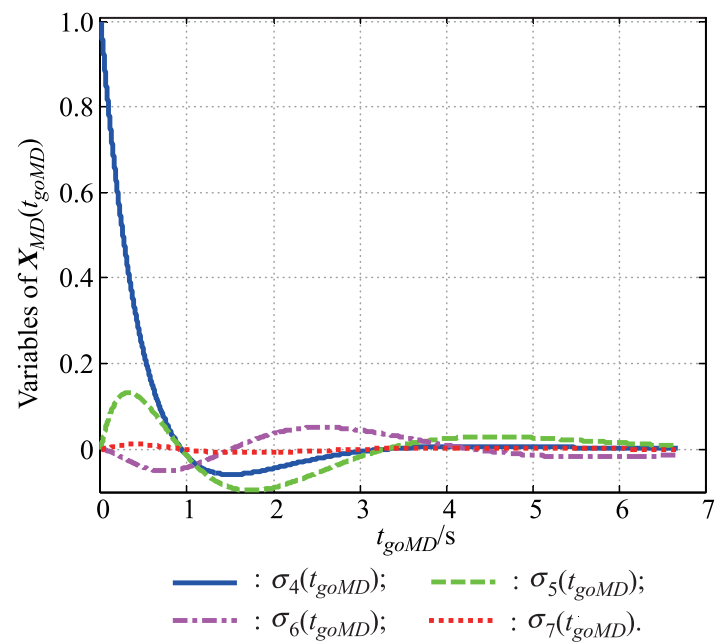

Fig. 12 Profiles of the vectors of $X_{M D}\left(t_{g o M D}\right)$ without control saturations
Fig. 13 and Fig. 14 demonstrate the profiles of zeroeffort miss distances $Z_{M T}(t)$ and $Z_{M D}(t)$ without control saturations respectively.

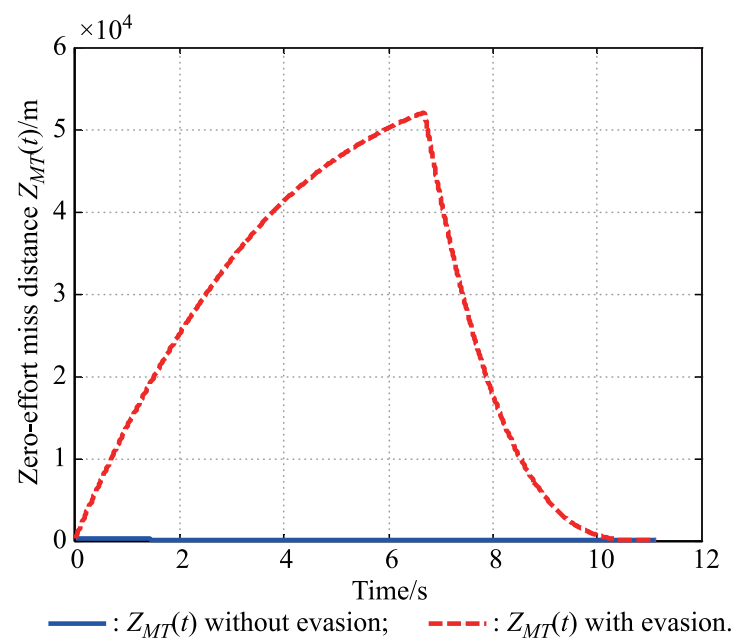

Fig. 13 Profiles of the zero-effort miss $Z_{M T}(t)$ without control saturations

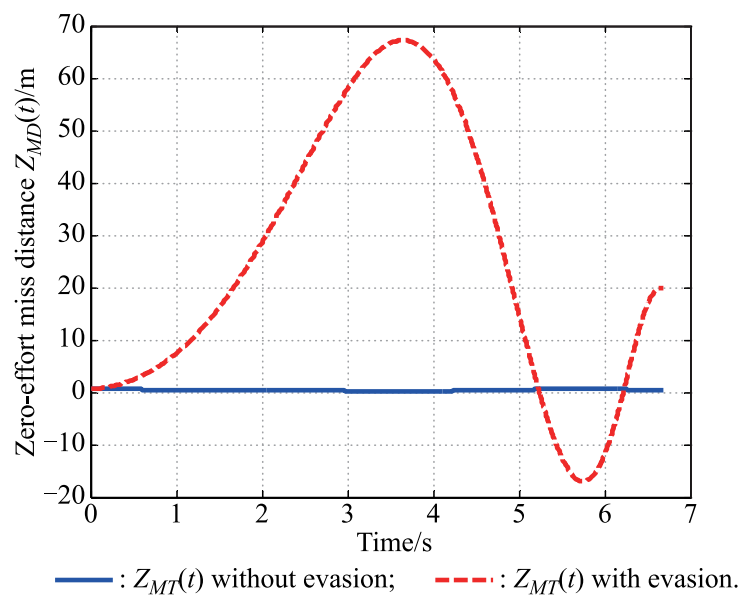

Fig. 14 Profiles of the zero-effort miss $Z_{M D}(t)$ without control saturations

From Fig. 13 and Fig. 14, it could be seen that the sacrifice of $Z_{M T}(t)$ for evading the defender is dramatically large.

Fig. 15 shows the calculated control profile without control saturations.

It seems that the results from Fig. 11 and Fig. 12 are reasonable and the optimal problem without control saturations can be solved. However, from Fig. 15, the control variables are pretty small when ignoring the coming defender but become significantly large. The control commands are far from practical implementation. Thus, the control saturations are necessary to get more convincing insight and conclusions. 


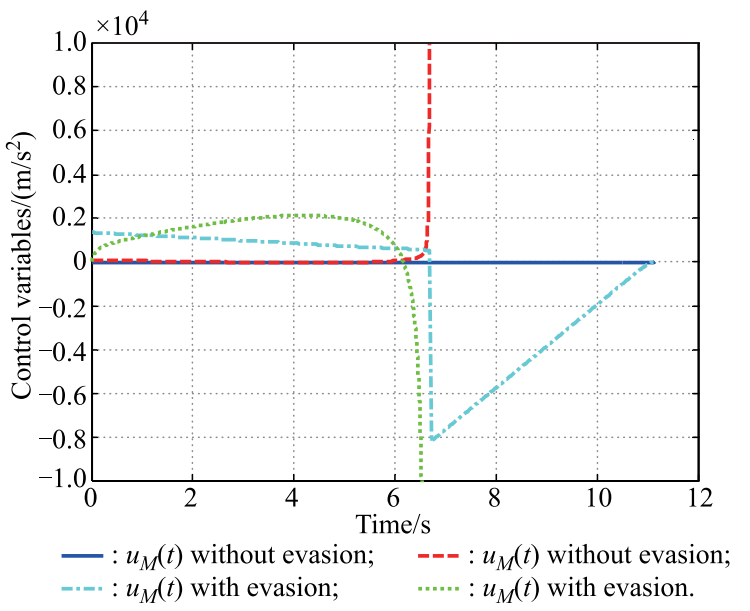

Fig. 15 Control profiles of the homing missile without control saturations

Then the simulation results with control saturations are expressed as follows. Fig. 16 and Fig. 17 illustrate the vectors of $\boldsymbol{X}_{M T}\left(t_{g o M T}\right)$ and $\boldsymbol{X}_{M D}\left(t_{g o M D}\right)$, respectively.

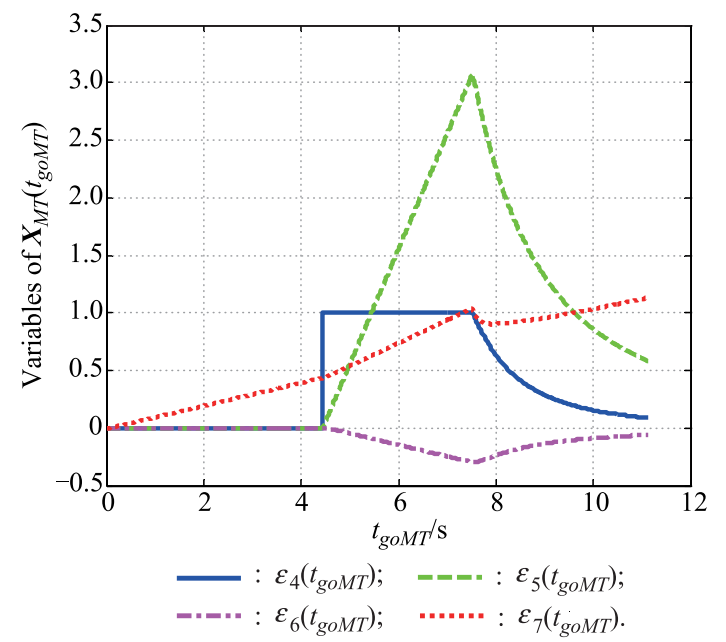

Fig. 16 Profiles of the vectors of $X_{M T}\left(t_{g o M T}\right)$ with control saturations

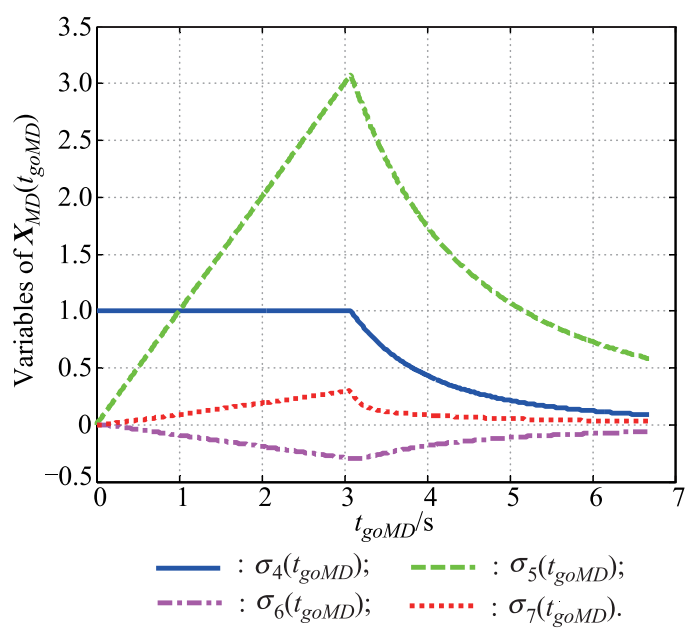

Fig. 17 Profile of the vectors of $X_{M D}\left(t_{g o M D}\right)$ with control saturations
It can be concluded that when the saturation occurrs, the variation of $\boldsymbol{X}_{M T}\left(t_{g o M T}\right)$ and $\boldsymbol{X}_{M D}\left(t_{g o M D}\right)$ is linear with the time-to-goes $t_{g o M T}$ and $t_{g o M D}$, respectively.

Fig. 18 and Fig. 19 present the profiles of the zero-effort miss distances $Z_{M T}(t)$ and $Z_{M D}(t)$, respectively.

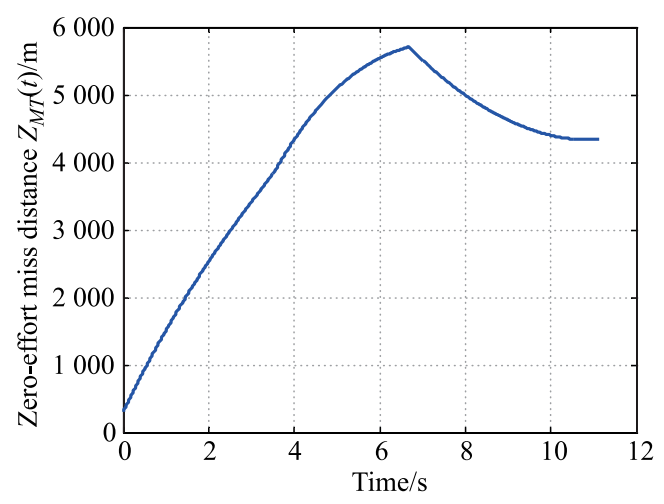

Fig. 18 Profile of the zero-effort miss $Z_{M T}(t)$ with control saturations

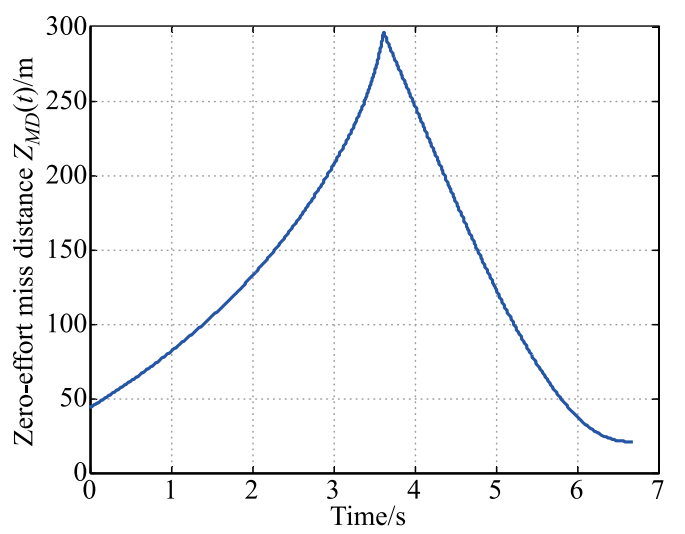

Fig. 19 Profile of the zero-effort miss $Z_{M D}(t)$ with control saturations

For the linearized model, the terminal value of $Z_{M T}(t)$ is still very large. However, the situation is reasonable, because the error arisen from the linearization has some impact on the terminal time $t_{f M T}$. In other words, the whole duration is extended due to the significant maneuver of the three agents. Thus the $Z_{M T}\left(t_{f M T}\right)$ or $y_{M T}\left(t_{f M T}\right)$ in the linearized model is not the terminal value due to the error of $t_{f M T}$. With regarding to $Z_{M D}(t)$, the proposed guidance algorithm is adequate to guarantee the required miss distance $M_{M D}$.

Fig. 20 demonstrates the open loop control profiles of the three agents in the linearized model. It could be found that the homing missile are required to maneuver by its maximum acceleration after evading the defender, which is caused by the significant sacrifice for the miss distance $Z_{M T}\left(t_{f M T}\right)$. 


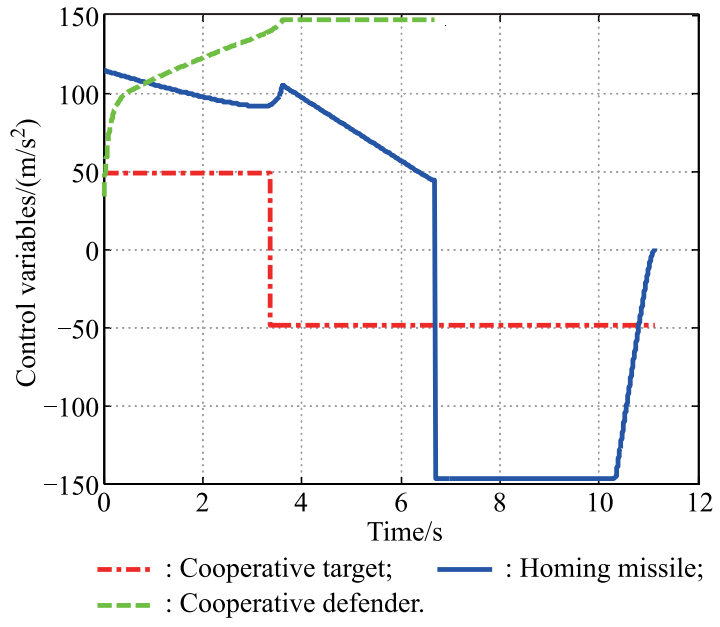

Fig. 20 Open loop control profiles of $T, M$ and $D$ with control saturations in linearized model

The proposed algorithm is eventually evaluated by numerical simulation in a nonlinear kinematics form. Fig. 21 presents the typical trajectories scenario of the TMD problem and Fig. 22 shows the corresponding control profiles of the three agents. Notice that the maneuver of target keeps in the duration $\left[0, t_{f M T}\right]$ merely, the same as the assumption in the linearized model.

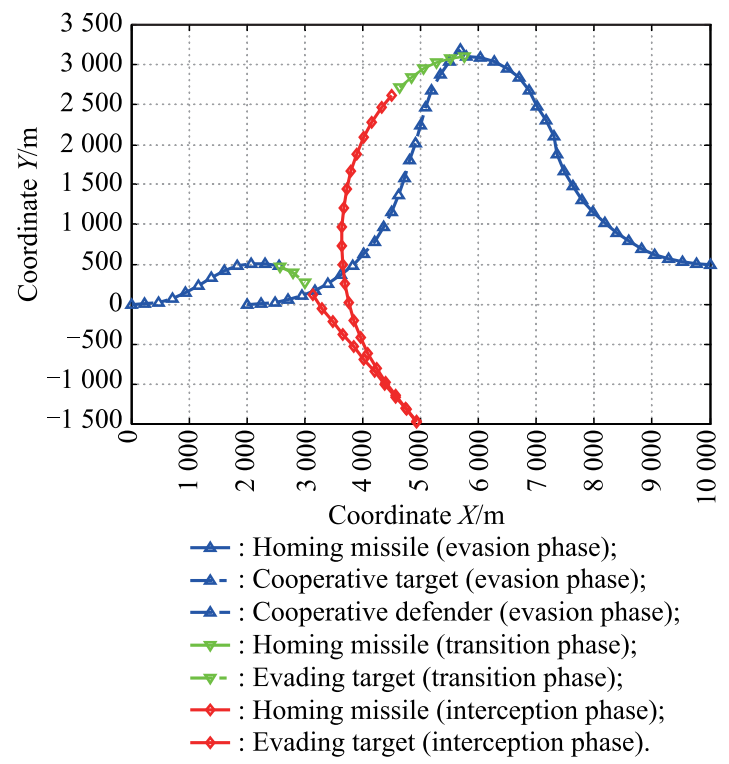

Fig. 21 Trajectory profiles of the TMD problem with control saturations and nonlinear kinematics

In Fig. 21, the whole process is divided into three phases:

(i) The evasion phase (the blue colored part). It corresponds to the time period $\left[0, t_{g o M D}\right]$ and the homing missile must evade the defender firstly. A relatively large maneuver is applied to the defender's control saturation and facilitate successful evasion.

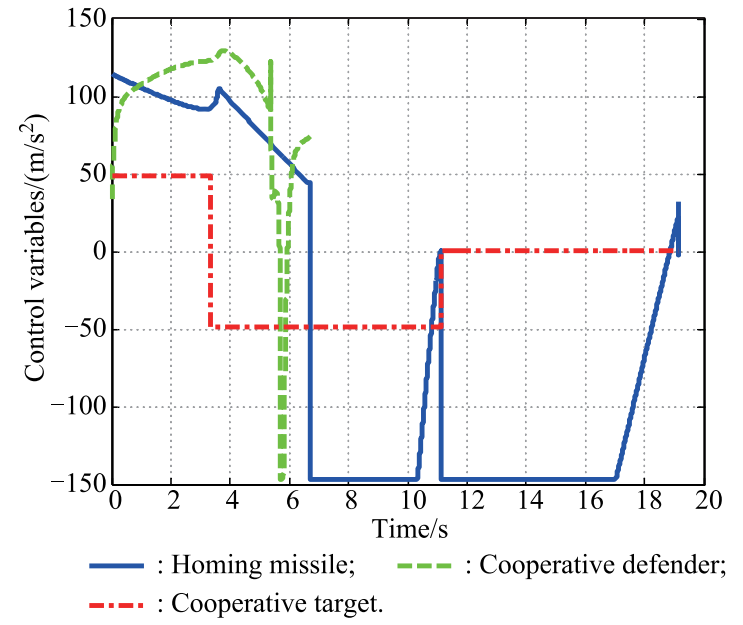

Fig. 22 Control profiles of the three agents with control saturations and nonlinear kinematics

(ii) The transition phase (the green colored part). It corresponds to the time period $\left[t_{g o M D}, t_{g o M T}\right]$ and the homing missile utilizes the maximum acceleration to swerve and intercept the target. Since the parameters such as $\alpha(t)$ arise from the linearized model, the control command approaches to zero at $t=t_{g o M T}$ due to the minimum energy part in the performance index.

(iii) The interception phase (the red colored part). It arises from the fact that in nonlinear kinematics, the whole engagement is extended. During this phase, the problem degenerates to a one-on-one pursuit-evasion game.

Consequently, in the model of nonlinear kinematics, the whole process is extended to $19.165 \mathrm{~s}$ with regarding to the terminal time $t_{f M T}=11.139 \mathrm{~s}$. Besides, the final miss distance between the defender and the homing missile is $121.69 \mathrm{~m}$ while that between the homing missile and the target is $0.42 \mathrm{~m}$.

In addition, comparisons with the ODGG and CMEG are conducted and the results are demonstrated as follows. The trajectories of the three agents in the time duration $\left[0, t_{f M T}\right]$ are illustrated in Fig. 23 and the accelerations of them in the time duration $\left[0, t_{f M D}\right]$ are demonstrated in Fig. 24.

It can be concluded that:

(i) When the homing missile utilizes the smart optimal guidance (M-SOG), the homing missile makes full use of its maneuverability at the beginning. This maneuver brings about the sharp increase of the defender's acceleration command. Then the homing missile's acceleration command decreases gradually, but still enough to produce large miss distance related to the defender.

(ii) When the homing missile utilizes the optimal differential game guidance (M-ODGG), it combines the two miss distances $y_{M T}\left(t_{f M T}\right)$ and $y_{M D}\left(t_{f M D}\right)$ all the time, 
and its acceleration command switches once during the evasion process from the defender. The zero-miss effort $Z_{M T}(t)$ increases so significantly that it leads to the change of the signal of the acceleration command. However, this switch exerts negative effects on the final miss distance $y_{M D}\left(t_{f M D}\right)$.

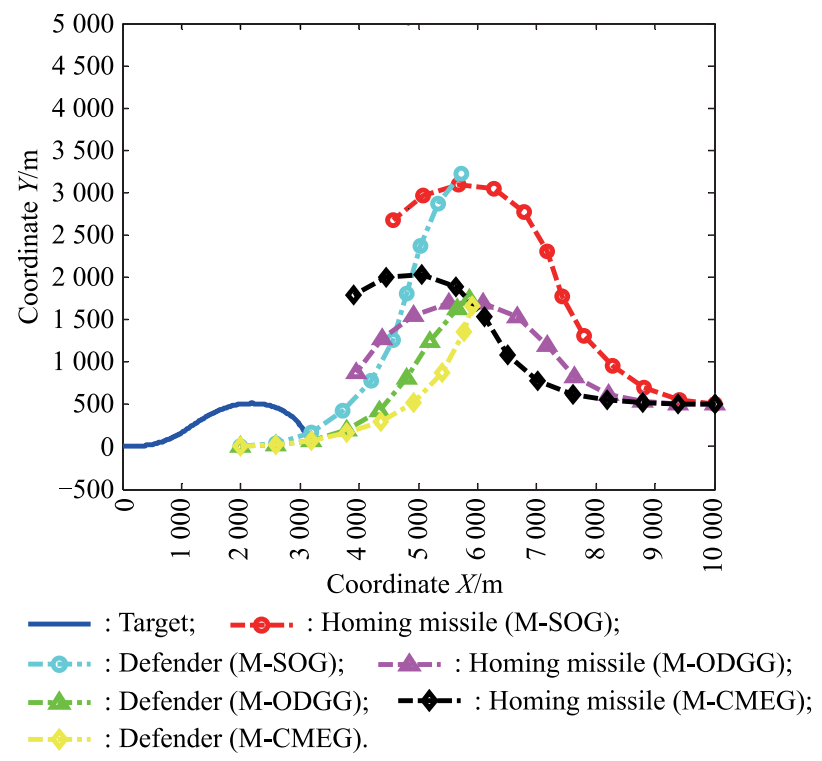

Fig. 23 Trajectory profiles comparison of the three agents under different guidance strategies

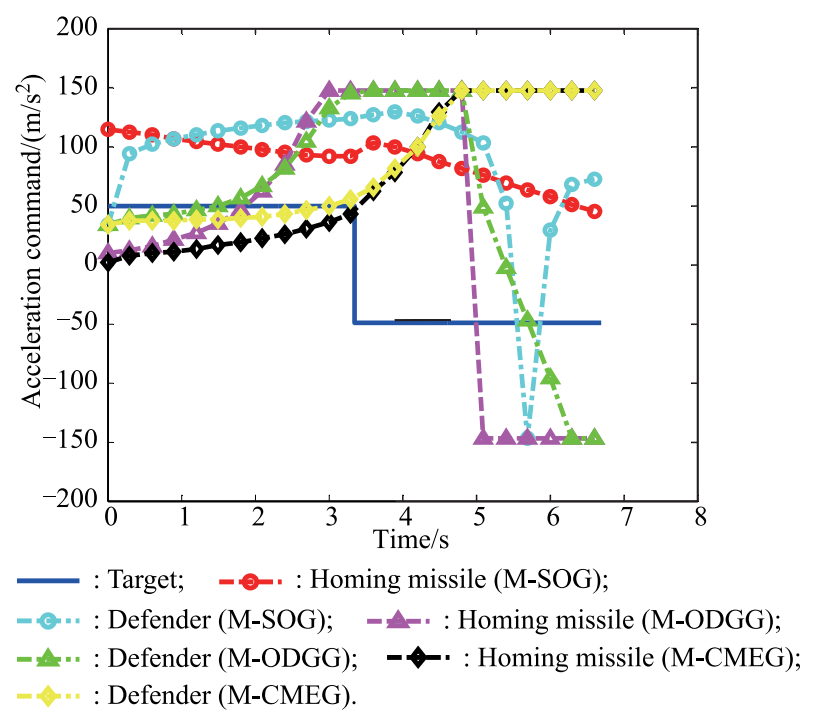

Fig. 24 Control profiles comparison of the three agents under different guidance strategies

(iii) When the homing missile utilizes the combined minimum effort guidance (M-CMEG), it is conservative at the beginning and the acceleration command saturates at last. In addition, the final miss distance $y_{M D}\left(t_{f M D}\right)$ is produced mostly due to the tail-pursuit engagement. In detail, the defender and the missile possess the same velo- city and at the endgame phase, the homing missile maneuvers construct a tail-pursuit scenario and thus the miss distance is acceptable. However, if the defender owns the velocity advantage, the homing missile will be intercepted probably.

The actual miss distances related to the defender are $121.21 \mathrm{~m}$ (M-SOG), $35.38 \mathrm{~m}$ (M-ODGG) and $95.31 \mathrm{~m}$ (MCMEG), respectively.

In addition to the comparisons experiments with other guidance laws, the performance of the proposed guidance algorithm is further investigated from the statistics point of view. A Monte-Carlo simulation is conducted with 500 experiments, and the initial position in $Y$ direction of the homing missile is chosen as the random variable, which varies from -1000 to 1000 in a uniform distribution manner. The average values of miss distances $y\left(t_{f M D}\right)$ and $y\left(t_{f M T}\right)$ are demonstrated in Table 3.

Table 3 Average values of the miss distances by Monte-Carlo simulations

\begin{tabular}{cccc}
\hline \multirow{2}{*}{ Parameter } & \multicolumn{3}{c}{ Value/m } \\
\cline { 2 - 4 } & SOG & ODGG & CMEG \\
\hline$y\left(t_{f M D}\right)$ & 73.01 & 21.64 & 60.72 \\
$y\left(t_{f M T}\right)$ & 0.34 & 0.25 & 0.29 \\
\hline
\end{tabular}

By the Monte-Carlo simulation, it can be validated that the SOG algorithm exhibites more desirable performance in the aspect of the miss distances.

On the whole, the SOG strategy guarantees the successful evasion from the cooperative defender and saves control energy to some extent. It turns out to be more reliable for practical engineering applications.

\section{Conclusions}

In this paper, a smart homing guidance strategy against a cooperative target-defender team is derived with a certain assumption on the cooperative strategy of the team. Firstly, the theoretically solution of the smart guidance algorithm is obtained based on the linearized model without control saturations.

However, in more practical situations, the efficient guidance law of the cooperative strategy of the target-defender team leads to the fact that the theoretical solution of the smart missile's acceleration command is too large to be implemented. Therefore, the nonlinear factor of control saturations is introduced for further reasonable insight of the TMD problem. Besides, the corresponding algorithm as well as the iterative searching technique to determine the saturation time points is proposed.

Eventually, by comparison with the ODGG and CMEG, it guarantees successful evasion againsts the defender with 
superior reliability and intercepts the target with high precision. The conclusions are aligned with the results of the Monte-Carlo simulation as well. Besides, the integral evasion-interception process of the smart homing missile is conducted under control saturations and in a minimum effort sense.

\section{References}

[1] RUSNAK I. The lady, the bandits and the body-guards - a two team dynamic game. Proc. of the 16th IFAC World Congress, 2005: $441-446$.

[2] BEN-ASHER J, CLIFF M E, KELLEY J H. Optimal evasion with a path-angle constraint and against two pursuers. Journal of Guidance, 1987, 11(4): 300-304.

[3] ONG Y S, PIERSON L B. Optimal planar evasive aircraft maneuvers against proportional navigation missiles. Journal of Guidance, Control and Dynamics, 19(6): 1210-1215.

[4] SHAFERMAN V, SHIMA T. Linear quadratic differential games guidance law for imposing a terminal intercept angle. Proc. of the AIAA Guidance, Navigation and Control Conference and Exhibit, 2008: 18-21.

[5] BARDHAN R, GHOSE D. Nonlinear differential games-based impact-angle-constrained guidance law. Journal of Guidance, Control and Dynamics, 2015, 38(3): 384-402.

[6] SHAFERMAN V, SHIMA T. Linear quadratic guidance laws for imposing a terminal intercept angle. Journal of Guidance, Control, and Dynamics, 2008, 31(5): 1400-1412.

[7] SINGH L. Autonomous missile avoidance using nonlinear model predictive control. Proc. of the AIAA Guidance, Navigation, and Control Conference and Exhibit, 2004: 16-19.

[8] KANG S, KIM H J. Aerial pursuit-evasion game using nonlinear model predictive guidance. Proc. of the AIAA Guidance, Navigation, and Control Conference, 2010: 2-5.

[9] FONOD R, SHIMA T. Multiple model adaptive evasion against a homing missile. Journal of Guidance, Control and Dynamics, 2016, 39(7): $1578-1592$.

[10] GARCIA E, CASBEER W D, FUCHS E Z, et al. Cooperative missile guidance for active defense of air vehicles. IEEE Trans. on Aerospace and Electronic System, 2018, 54(2): 706-721.

[11] KUMAR S R, SHIMA T. Cooperative nonlinear guidance strategies for aircraft defense. Journal of Guidance, Control and Dynamics, 2017, 40(1): 124-138.

[12] GARCIA E, CASBEER W D, PACHTER M. Optimal guidance for active aircraft defense against homing missiles. Proc. of the AIAA Guidance, Navigation and Control Conference, 2017: 9-13.

[13] SHALUMOV V, SHIMA T. Weapon-target-allocation strategies in multiagent target-missile-defender engagement. Journal of Guidance, Control and Dynamics, 2017, 40(10): $2452-$ 2464.

[14] PROKOPOV O, SHIMA T. Linear quadratic optimal cooperative strategies for active aircraft protection. Journal of Guidance, Control and Dynamics, 2013, 36(3): 753-764.

[15] GARCIA E, CASBEER D W, PACHTER M. Cooperative strategies for optimal aircraft defense from an attacking missile. Journal of Guidance, Control and Dynamics, 2015, 38(8): $1510-1520$.

[16] SHAFERMAN V, SHIMA T. A cooperative differential game for imposing a relative intercept angle. Proc. of the AIAA
Guidance, Navigation, and Control Conference, 2017: 9-13.

[17] BALHANCE N, WEISS M, SHIMA T. Cooperative guidance law for intrasalvo tracking. Journal of Guidance, Control and Dynamics, 2017, 40(6): $1441-1456$.

[18] SHAFERMAN V, SHIMA T. Cooperative multiple model adaptive guidance for an aircraft defending missile. Journal of Guidance, Control and Dynamics, 2010, 33(6): 1801-1813.

[19] RUBINSKY S, GUTMAN S. Three-player pursuit and evasion conflict. Journal of Guidance, Control and Dynamics, 2014, 37(1): $98-110$.

[20] PERELMAN A, SHIMA T, RUSNAK I. Cooperative differential games strategies for active aircraft protection from a homing missile. Journal of Guidance, Control and Dynamics, 2011, 34(3): $761-773$.

[21] WEISS M, SHIMA T, CASTANEDA D, et al. Combined and cooperative minimum-effort guidance algorithms in an active aircraft defense scenario. Journal of Guidance, Control and Dynamics, 2017, 40(5): 1241 - 1254.

[22] WEISS M, SHIMA T, CASTANEDA D. Minimum effort intercept and evasion guidance algorithms for active aircraft defense. Journal of Guidance, Control and Dynamics, 2016, 39(10): $2297-2311$.

[23] TAN Z W, FONOD R, SHIMA T. Cooperative guidance law for target pair to lure two pursuers into Collision. Journal of Guidance, Control and Dynamics, 2018, 41(8): 1687-1699.

[24] HU Z H, ZHOU D. Differential game guidance law considering second-order dynamics with zeros of missile autopilots. Proc. of the 36th Chinese Control Conference, 2017: 26-28.

[25] SUN W, TSIOTRAS P, LOLLA T, et al. Multiple-pursuer/oneevader pursuit-evasion game in dynamic flowfields. Journal of Guidance, Control and Dynamics, 2017, 40(7): 1627-1637.

[26] YANG Z, ZHOU D Y, PAN Q, et al. Research on adaptive robust guidance law for passive homing missile against maneuvering target. Proc. of the IEEE International Conference on Information and Automation, 2017: 527-530.

[27] TURETSKY V, SHIMA T. Target evasion from a missile performing multiple switches in guidance law. Journal of Guidance, Control and Dynamics, 2016, 39(10): 2364-2373.

[28] FONOD R, SHIMA T. Target evasion strategy against a finite set of missile guidance laws. Proc. of the European Control Conference, 2016: 655-660.

[29] SUNKARA V, CHAKRAVARTHY A, GHOSE D. Pursuit evasion games using collision cones. Proc. of the AIAA Guidance, Navigation, and Control Conference, 2018: 1-16.

[30] LYU S, ZHU H Z, TANG S, et al. Hybrid cooperative guidance law for active aircraft defense against a guided missile. Journal of Guidance, Control and Dynamics, 2018, 41(2): 531 - 537.

[31] CARR W R, COBB G R, PACHTER M, et al. Solution of a pursuit-evasion game using a near-optimal strategy. Journal of Guidance, Control and Dynamics, 2018, 41(4): 841-850.

[32] WEI X Q, YANG J Y. Optimal strategies for multiple unmanned aerial vehicles in a pursuit/evasion differential game. Journal of Guidance, Control and Dynamics, 2018, 41(8): $1798-1805$.

[33] LIVERMORE R, SHIMA T. Deviated pure-pursuit-based optimal guidance law for imposing intercept time and angle. Journal of Guidance, Control and Dynamics, 2018, 41(8): $1805-1812$.

[34] ZOU X G, ZHOU D, DU R L, et al. Active defense guidance law via cooperative identification and estimation. Journal of Guidance, Control and Dynamics, 2018, 41(11): 2503-2511. 


\section{Biographies}

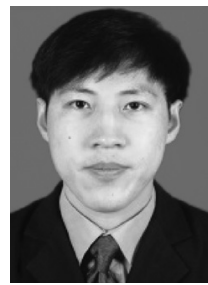

GUO Hang was born in 1990. He received his B.S. degree from Northwestern Polytechnical University in 2011. He received his M.S. degree from School of Astronautics, Northwestern Polytechnical University in 2014. He is a Ph.D. candidate with School of Astronautics, Northwestern Polytechnical University. His research interests are hypersonic vehicle's guidance and control and flight dynamics.

E-mail: jsguoh@mail.nwpu.edu.cn

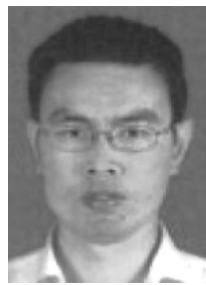

FU Wenxing was born in 1974. He received his Ph.D. degree from School of Astronautics, Northwestern Polytechnical University in 2006. As a professor, he is working in the School of Astronautics, Northwestern Polytechnical University. His research interests are system simulation and aircraft design. E-mail: wenxingfu@nwpu.edu.cn

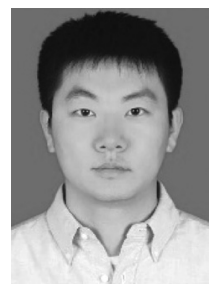

FU Bin was born in 1989. He received his B.S. degree from Northwestern Polytechnical University in 2011. He received his M.S. degree from School of Astronautics, Northwestern Polytechnical University in 2014. He is now a Ph.D. candidate with the School of Astronautics, Northwestern Polytechnical University. His research interests are guidance, navigation and control.

E-mail: binfu@mail.nwpu.edu.cn

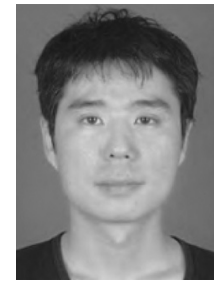

CHEN Kang was born in 1980. He received his Ph.D. degree from School of Astronautics Northwestern Polytechnical University in 2010. As an associate professor, he is working in the School of Astronautics, Northwestern Polytechnical University. His research interests are flight control, guidance and aircraft design.

E-mail: mars_legend@163.com

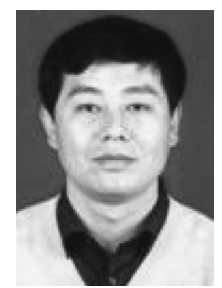

YAN Jie was born in 1960. He received his Ph.D. degree from School of Astronautics, Northwestern Polytechnical University in 1988. He is currently working as a professor and Ph.D. candidate supervisor in the School of Astronautics, Northwestern Polytechnical University. His research interests are flight control, guidance, system simulation and aircraft design.

E-mail: jyan@nwpu.edu.cn 